\title{
Bi-Maxwellian, slowing-down, and ring velocity distributions of fast ions in magnetized
} plasmas

\author{
Moseev, Dmitry; Salewski, Mirko
}

Published in:

Physics of Plasmas

Link to article, DOI:

$10.1063 / 1.5085429$

Publication date:

2019

Document Version

Publisher's PDF, also known as Version of record

Link back to DTU Orbit

Citation (APA):

Moseev, D., \& Salewski, M. (2019). Bi-Maxwellian, slowing-down, and ring velocity distributions of fast ions in magnetized plasmas. Physics of Plasmas, 26(2). https://doi.org/10.1063/1.5085429

\section{General rights}

Copyright and moral rights for the publications made accessible in the public portal are retained by the authors and/or other copyright owners and it is a condition of accessing publications that users recognise and abide by the legal requirements associated with these rights.

- Users may download and print one copy of any publication from the public portal for the purpose of private study or research.

- You may not further distribute the material or use it for any profit-making activity or commercial gain

- You may freely distribute the URL identifying the publication in the public portal

If you believe that this document breaches copyright please contact us providing details, and we will remove access to the work immediately and investigate your claim 


\title{
Bi-Maxwellian, slowing-down, and ring velocity distributions of fast ions in magnetized plasmas
}

Cite as: Phys. Plasmas 26, 020901 (2019); doi: 10.1063/1.5085429

Submitted: 12 December 2018 - Accepted: 4 February 2019 .

Published Online: 27 February 2019
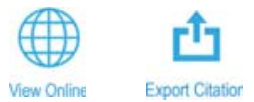

Dmitry Moseev ${ }^{1, a)}$ and Mirko Salewski ${ }^{2, b)}$ (iD

\section{AFFILIATIONS}

${ }^{1}$ Max-Planck-Institut für Plasmaphysik, Wendelsteinstr. 1, 17491 Greifswald, Germany

${ }^{2}$ Department of Physics, Technical University of Denmark, Fysikvej, Building 309, 2800 Kongens Lyngby, Denmark

a)dmmo@ipp.mpg.de

b) msal@dtu.dk

\begin{abstract}
We discuss analytical fast-ion velocity distribution functions which are useful for basic plasma modelling as illustrated for the tokamak ITER. The Maxwellian is by far the most widespread model for ions and electrons in tokamaks and stellarators. The biMaxwellian and the drifting (bi-)Maxwellian are extensions allowing for anisotropy and bulk plasma flow, respectively. For example, fast ions generated by wave heating in the ion cyclotron range of frequencies are often described by bi-Maxwellians or socalled tail temperatures. The ring distribution can serve as a basic building block for arbitrary distributions or as a bump-on-tail in stability studies. The isotropic slowing-down distribution is a good model for fusion $\alpha$-particles. The anisotropic slowing-down distribution occurs for anisotropic particle sources as is typical for neutral beam injection. We physically motivate these distribution functions and present analytical models in various coordinate systems commonly used by theorists and experimentalists. We further calculate $1 \mathrm{D}$ projections of the distribution functions onto a diagnostic line-of-sight to gain insight into measurements relying on the Doppler shift.
\end{abstract}

Published under license by AIP Publishing. https://doi.org/10.1063/1.5085429

\section{INTRODUCTION}

Probably, the most widely known velocity distribution function of particles in all of physics is the Maxwellian or MaxwellBoltzmann distribution function which is on the curriculum of most physics educations, see, e.g., Refs. 1-3. Maxwell's groundbreaking idea was to describe the state of a gas by a distribution function $f(\mathbf{v})$ of velocities $\mathbf{v}$. In his 1860 paper, ${ }^{4}$ he introduced his distribution based on two requirements. First, the function should be spherically symmetric reflecting isotropy. Second, the velocity components should be separable reflecting the independence of the three coordinate directions. The Maxwell distribution is the only distribution fulfilling both requirements. (However, the velocity components are not separable if relativistic effects are considered. ${ }^{3}$ ) In 1867, he showed without reference to his two original requirements that collisions do not change his distribution. ${ }^{5}$ Boltzmann showed in the following decade that the Maxwellian is the most probable distribution in an isolated system with a given particle number and a given energy and that collisions will drive any distribution towards it, i.e., that it is the only stationary solution. ${ }^{6,7}$ A collection of particles described by the Maxwellian distribution is said to be in thermal equilibrium which is an excellent approximation for rarefied gases at moderate temperatures.

However, plasmas in tokamaks and stellarators are never in complete thermal equilibrium. Fusion plasmas are much hotter than the environment and lose heat rapidly. This heat loss must be balanced by a heat source from fusion reactions or from auxiliary heating, which prevents the formation of a thermal equilibrium even in a steady-state plasma. For example, the velocity distribution function of fusion $\alpha$-particles has a tail approximately of the form $1 / v^{3}$ up to the birth velocity rather than a Maxwellian tail. ${ }^{8-10}$ The energetic particle populations originating from neutral beam injection (NBI) and electromagnetic wave heating in the ion cyclotron range of frequencies (ICRFs) are even highly anisotropic in velocity space..$^{11-15}$ Nevertheless, Maxwellians have often been assumed to approximate the bulk plasma with success.

This tutorial reviews the Maxwellian distribution as well as velocity distribution functions typical for plasma scenarios with fusion or auxiliary heating: the drifting Maxwellian and 
bi-Maxwellian distributions, the ring distribution, and the isotropic and the anisotropic slowing-down distributions. In each case, we discuss the $1 \mathrm{D}$ projection of the distribution which is highly important for diagnostics viewing along a characteristic direction such as the line-of-sight. These analytical velocity distribution functions have many applications: to quickly model plasma discharges, to interpret measurements with fusion plasma diagnostics, to make theoretical investigations and computer codes tractable, or to benchmark computer codes. They can serve as the first step to get an overview of a discharge before turning to high-fidelity codes, and they can aid diagnostic design in new tokamaks or stellarators where the exact distribution function may not yet be known.

We will introduce these common velocity distribution functions in the most widespread coordinate systems in plasma physics. It is often not explicitly stated or obvious what exactly a distribution function refers to. Experimentalists tend to consider fully transformed distribution functions which include all Jacobians and normalization factors. Many theorists, however, tend to simply substitute convenient variables into a 3D Cartesian velocity distribution function but not actually transform it to the new variables.

Fully 3D functions are described in 3D Cartesian, cylindri$\mathrm{cal}$, and spherical coordinates. Velocity distribution functions in magnetized fusion plasmas are axisymmetric about the magnetic field vector (sometimes called gyrotropic) to a good approximation due to the fast gyration of the charged particles. Axisymmetric functions can be described by two coordinates which are often chosen to be 2D Cartesian or (E, $p)$-coordinates. $E$ is the energy, and

$$
p=\frac{\mathbf{v} \cdot \mathbf{B}}{|\mathbf{v}||\mathbf{B}|}
$$

is the pitch of the particle, where $\mathbf{v}$ and $\mathbf{B}$ are the velocity and magnetic field vectors, respectively. (This definition may sometimes have a minus sign if the magnetic field and the current point are in opposing directions so that co-current passing ions have a positive pitch.) Sometimes, distributions are assumed to be isotropic as, for example, the Maxwellian according to Maxwell's 1860 requirement. ${ }^{4}$ Isotropic functions can be described by just one coordinate, commonly, the speed or the energy. Finally, the distribution of projected velocities onto a particular direction is another important 1D description for many diagnostics. The underlying velocity distribution is usually anisotropic to some degree. Hence, some information is lost in the projection.

This paper is organized as follows: Section II introduces the various coordinate systems used in fusion plasma physics. Section III transforms the Maxwellian to these coordinate systems. Section IV discusses the drifting bi-Maxwellian distribution and Sec. $V$ the topologically different ring distribution which is described by a very similar equation. Section VI reviews the slowing down of particles with isotropic and anisotropic particle sources. Section VII briefly outlines a model for the global phase-space distribution function parametrized in three constants of motion of a particle. Section VIII concludes this tutorial.

\section{DISTRIBUTION FUNCTIONS IN VARIOUS COORDINATES}

In general, phase-space distribution functions are sixdimensional, consisting of three position-space dimensions and three velocity-space dimensions. The phase-space distribution $f^{6 \mathrm{D}}(\mathbf{x}, \mathbf{v})$ specifies the number of particles $d \mathrm{~N}$ in an infinitesimal phase-space volume $(d \mathbf{x}, d \mathbf{v})$

$$
d \mathrm{~N}=f^{6 \mathrm{D}}(\mathbf{x}, \mathbf{v}) d \mathbf{x} d \mathbf{v}
$$

The unit of $f^{6 D}$ is $s^{3} / m^{6}$. In this tutorial, we consider local velocity distribution functions such that only the three velocity-space dimensions are relevant. The velocitydistribution function in the infinitesimal position-space volume element $d \mathbf{x}$ is defined by

$$
d \mathrm{~N}=f^{3 \mathrm{D}}(\mathbf{v}) d \mathbf{v} d \mathbf{x}
$$

Dividing both sides by $d \mathbf{x}$ introduces the density $d n$ in the infinitesimal velocity-space volume $d \mathbf{v}$

$$
d n=f^{3 \mathrm{D}}(\mathbf{v}) d \mathbf{v} .
$$

The unit of $f^{3 \mathrm{D}}$ is also $\mathrm{s}^{3} / \mathrm{m}^{6}$. The density is obtained by integration which provides the normalization condition for the distribution function

$$
n=\int f^{3 \mathrm{D}}(\mathbf{v}) d \mathbf{v}
$$

Various representations of distribution functions are commonly used in the plasma physics literature. We first consider convenient 3D Cartesian velocity coordinates for fusion plasmas. Particles in magnetized plasmas gyrate quickly about the magnetic field vector so that velocity distribution functions are axisymmetric about $\mathbf{B}$ to a good approximation. Hence, it is advantageous to align one of the coordinate axes with the magnetic field vector. This axis is called $v_{\|} \cdot v_{\perp 1}$ and $v_{\perp 2}$ are the velocity components perpendicular to the magnetic field. The velocity distribution function $f_{\text {Car }}^{3 \mathrm{D}}\left(v_{\|}, v_{\perp 1}, v_{\perp 2}\right)$ gives the density in an infinitesimal velocityspace volume

$$
d n=f_{\text {Car }}^{3 \mathrm{D}}\left(v_{\|}, v_{\perp 1}, v_{\perp 2}\right) d v_{\|} d v_{\perp 1} d v_{\perp 2} .
$$

Axisymmetric functions do not depend on the gyrophase $\gamma$. Hence, it is advantageous to introduce the cylindrical coordinates $\left(v_{\|}, v_{\perp}, \gamma\right)$ such that

$$
\begin{aligned}
& v_{\perp 1}=v_{\perp} \cos \gamma, \\
& v_{\perp 2}=v_{\perp} \sin \gamma .
\end{aligned}
$$

$v_{\perp}$ is the perpendicular velocity, which is related to $v_{\perp 1}$ and $v_{\perp 2}$ by

$$
v_{\perp}=\sqrt{v_{\perp 1}^{2}+v_{\perp 2}^{2}} .
$$

Substitution of $v_{\perp 1}$ and $v_{\perp 2}$ gives a convenient representation of $3 \mathrm{D}$ axisymmetric functions in two coordinates, $f_{\mathrm{Car}}^{3 \mathrm{D}}\left(v_{\|}, v_{\perp}\right)$, since the ignorable gyrophase $\gamma$ drops out. The density in an infinitesimal velocity-space volume is 


$$
d n=f_{\mathrm{Car}}^{3 \mathrm{D}}\left(v_{\|}, v_{\perp}\right) d v_{\|} d v_{\perp 1} d v_{\perp 2} .
$$

This substitution does not correspond to a transformation of the velocity distribution function to the cylindrical coordinates $\left(v_{\|}, v_{\perp} \cdot \gamma\right)$. The distribution function $f_{\mathrm{Car}}^{3 \mathrm{D}}\left(v_{\|}, v_{\perp}\right)$ still represents the 3D Cartesian velocity space density, but it is parametrized in cylindrical coordinates. The velocity distribution function could formally be written as $f_{\mathrm{Car}}^{3 \mathrm{D}}\left(v_{\|}, v_{\perp}\left(v_{\perp 1}, v_{\perp 2}\right)\right)$ to state the dependence of $v_{\perp}$ on the underlying Cartesian coordinates $v_{\perp 1}$ and $v_{\perp 2}$ [Eq. (9)], but this is not usually done. For a full transformation to cylindrical coordinates, we also need to express the velocity-space volume element in cylindrical coordinates. The Jacobian of the transformation from Cartesian to cylindrical coordinates is

$$
\mathrm{J}=\operatorname{det}\left|\frac{\partial\left(v_{\|}, v_{\perp 1}, v_{\perp 2}\right)}{\partial\left(v_{\|}, v_{\perp}, \gamma\right)}\right|=v_{\perp},
$$

so that the density in the small velocity-space volume is given by

$$
d n=f_{\mathrm{Car}}^{3 \mathrm{D}}\left(v_{\|}, v_{\perp}\right) v_{\perp} d v_{\|} d v_{\perp} d \gamma .
$$

Integration over $\gamma$ conveniently reduces the number of dimensions. The density is then given by

$$
d n=2 \pi v_{\perp} f_{\mathrm{Car}}^{3 \mathrm{D}}\left(v_{\|}, v_{\perp}\right) d v_{\|} d v_{\perp} .
$$

Distribution functions computed with the TRANSP/NUBEAM code ${ }^{16}$ which is implemented in most tokamaks, are usually presented in $(E, p)$-coordinates. This coordinate system is probably the most widespread among experimentalists working with velocity or distribution functions. The $2 \mathrm{D}$ coordinate transformations between $\left(v_{\|}, v_{\perp}\right)$ and $(\mathrm{E}, p)$ are

$$
\begin{aligned}
& \mathrm{E}=\frac{1}{2} m\left(v_{\|}^{2}+v_{\perp}^{2}\right), \quad v_{\|}=p \sqrt{\frac{2 \mathrm{E}}{m}}, \\
& p=\frac{v_{\|}}{\sqrt{v_{\|}^{2}+v_{\perp}^{2}}}, \quad v_{\perp}=\sqrt{1-p^{2}} \sqrt{\frac{2 \mathrm{E}}{m}},
\end{aligned}
$$

where $m$ is the particle mass. The definition of the pitch in Eq. (1) and the transformation in Eq. (14) show that the pitch $p \in[-1 ; 1]$. It is the cosine of the so-called pitch angle. The Jacobians for the forward and backward transformations are

$$
\begin{aligned}
& \mathrm{J}_{v_{\|}, v_{\perp} \rightarrow \mathrm{E}, p}=\operatorname{det}\left|\frac{\partial\left(v_{\|}, v_{\perp}\right)}{\partial(\mathrm{E}, p)}\right|=\frac{1}{m \sqrt{1-p^{2}}}, \\
& \mathrm{~J}_{\mathrm{E}, p \rightarrow v_{\|}, v_{\perp}}=\operatorname{det}\left|\frac{\partial(\mathrm{E}, p)}{\partial\left(v_{\|}, v_{\perp}\right)}\right|=\frac{m v_{\perp}}{\sqrt{v_{\|}^{2}+v_{\perp}^{2}}} .
\end{aligned}
$$

The density in the infinitesimal area $d E d p$ is

$$
d n=2 \pi \sqrt{\frac{2 \mathrm{E}}{m^{3}}} f_{\mathrm{Car}}^{3 \mathrm{D}}\left(p \sqrt{\frac{2 \mathrm{E}}{m}}, \sqrt{1-p^{2}} \sqrt{\frac{2 \mathrm{E}}{m}}\right) d \mathrm{E} d p .
$$

[However, the unit of the distribution in the actual output file of TRANSP is $1 / \mathrm{cm}^{3} / \mathrm{eV} /(d \Omega / 4 \pi)$, where $d \Omega$ is the solid angle. As $d \Omega=2 \pi d p, d \Omega / 4 \pi=d p / 2$ so that we have to divide the TRANSP output by two to obtain the distribution function in the often used $(E, p)$-coordinates.]
Sometimes, the speed is used instead of the energy. The speed is

$$
v=\sqrt{v_{\|}^{2}+v_{\perp 1}^{2}+v_{\perp 2}^{2}} .
$$

The $2 \mathrm{D}$ coordinate transformations between $\left(v_{\|}, v_{\perp}\right)$ and $(v, p)$ are

$$
\begin{array}{ll}
v=\sqrt{v_{\|}^{2}+v_{\perp}^{2}}, & v_{\|}=p v, \\
p=\frac{v_{\|}}{\sqrt{v_{\|}^{2}+v_{\perp}^{2}}}, \quad v_{\perp}=\sqrt{1-p^{2} v .}
\end{array}
$$

The Jacobians for forward and backward transformations between $\left(v_{\|}, v_{\perp}\right)$ and $(v, p)$ are

$$
\mathrm{J}_{v_{\|}, v_{\perp} \rightarrow v, p}=\frac{v}{\sqrt{1-p^{2}}}, \quad \mathrm{~J}_{v, p \rightarrow v_{\|}, v_{\perp}}=\frac{v_{\perp}}{v_{\|}^{2}+v_{\perp}^{2}} .
$$

The density in the infinitesimal area $d v d p$ is

$$
d n=2 \pi v^{2} f_{\mathrm{C} a r}^{3 \mathrm{D}}\left(p v, \sqrt{1-p^{2}} v\right) d v d p .
$$

One can also arrive at this result by transforming the density in $(\mathrm{E}, p)$-coordinates $[(\mathrm{Eq} .(16)]$ to $(v, p)$-coordinates. These coordinates are related by the usual definition of kinetic energy

$$
\mathrm{E}=\frac{1}{2} m v^{2}
$$

The Jacobians for forward and backward transformations between speed and energy are

$$
\mathrm{J}_{v \rightarrow \mathrm{E}}=\frac{d v}{d \mathrm{E}}=\frac{1}{\sqrt{2 m \mathrm{E}}}, \quad \mathrm{J}_{\mathrm{E} \rightarrow v}=\frac{d \mathrm{E}}{d v}=m v .
$$

Whereas gyrotropic functions are described by two variables, isotropic distribution functions are described by just one variable, most often the speed or the energy. If the spherical coordinates $(v, \eta, \zeta)$ are substituted into an isotropic distribution function, the two angles drop out and we can write the isotropic distribution function as $f_{\mathrm{Car}}^{3 \mathrm{D}}(v)$. This function represents a 3D Cartesian velocity space density parametrized in spherical coordinates. The density in the infinitesimal velocity-space volume element is

$$
d n=v^{2} \sin \zeta f_{\text {Car }}^{3 \mathrm{D}}(v) d v d \eta d \zeta,
$$

where $v^{2} \sin \zeta$ is the Jacobian of the transformation from Cartesian to spherical coordinates. As the isotropic function $f_{\mathrm{Car}}^{3 \mathrm{D}}(v)$ does not depend on the angles, the density can be expressed as a function of the speed alone by integration over the angles

$$
d n=4 \pi v^{2} f_{\text {Car }}^{3 \mathrm{D}}(v) d v .
$$

The energy is another popular coordinate. In terms of energy, we get

$$
d n=4 \pi \sqrt{\frac{2 \mathrm{E}}{m^{3}}} f_{\mathrm{Car}}^{3 \mathrm{D}}\left(\sqrt{\frac{2 \mathrm{E}}{m}}\right) d \mathrm{E} .
$$


The distribution functions transformed to these various coordinates can be related to the 3D Cartesian coordinate system. The prefactor contains the relevant Jacobian and a factor $2 \pi$ or $4 \pi$ from the integration over the angle or solid angle, respectively,

$$
\begin{gathered}
f_{c y l}^{3 \mathrm{D}}\left(v_{\|}, v_{\perp}\right)=v_{\perp} f_{\mathrm{Car}}^{3 \mathrm{D}}\left(v_{\|}, v_{\perp}\right), \\
f_{\mathrm{C} a r}^{2 \mathrm{D}}\left(v_{\|}, v_{\perp}\right)=2 \pi v_{\perp} f_{\mathrm{C} a r}^{3 \mathrm{D}}\left(v_{\|}, v_{\perp}\right), \\
f_{v p}^{2 \mathrm{D}}(v, p)=2 \pi v^{2} f_{\mathrm{Car}}^{3 \mathrm{D}}\left(p v, \sqrt{1-p^{2}} v\right), \\
f_{\mathrm{Ep}}^{2 \mathrm{D}}(\mathrm{E}, p)=2 \pi \sqrt{\frac{2 \mathrm{E}}{m^{3}}} f_{\mathrm{Car}}^{3 \mathrm{D}}\left(p \sqrt{\frac{2 \mathrm{E}}{m}}, \sqrt{1-p^{2}} \sqrt{\frac{2 \mathrm{E}}{m}}\right), \\
f_{v}^{1 \mathrm{D}}(v)=4 \pi v^{2} f_{\mathrm{Car}}^{3 \mathrm{D}}(v), \\
f_{\mathrm{E}}^{1 \mathrm{D}}(\mathrm{E})=4 \pi \sqrt{\frac{2 \mathrm{E}}{m^{3}}} f_{\mathrm{Car}}^{3 \mathrm{D}}\left(\sqrt{\frac{2 \mathrm{E}}{m}}\right) .
\end{gathered}
$$

Velocity or energy distribution functions common in the plasma physics literature may or may not include the factor $2 \pi$ or $4 \pi$ from the integration over ignorable angle(s) and may or may not include the Jacobian from Cartesian coordinates to various coordinate systems. As usually the coordinate system or the infinitesimal volume element is not explicitly stated, it may not be immediately obvious which coordinate system an author has in mind. Theorists will often work with the 3D Cartesian velocity distribution function parametrized in cylindrical coordinates. Experimentalists will often prefer to include the Jacobian and the integral over the ignorable angle(s) as implemented in the widespread TRANSP/NUBEAM code. Inclusion of all terms is also necessary to find the distribution function in these variables. For example, the 1D Maxwellian speed distribution and the Maxwellian energy distributions do contain the relevant Jacobians and the factor $4 \pi$ in the vast majority of textbooks on basic physics and statistical physics, e.g., Ref. 2.

In diagnostic applications, we are often interested in velocity distribution functions projected onto one particular direction, often given by the line-of-sight of the diagnostic. A few examples are charge-exchange recombination spectroscopy including fast-ion $\mathrm{D}_{\alpha}$-spectroscopy, Thomson scattering and collective Thomson scattering, neutron emission spectroscopy, and $\gamma$-ray spectroscopy. ${ }^{17}$ The projection of isotropic functions is independent of the direction and can hence be found by integration of $f^{3 \mathrm{D}}(\mathbf{v})$ over two coordinates as often shown in textbooks. An arbitrary function in $3 \mathrm{D}$ velocity space is in general projected according to

$$
g(u)=\int_{-\infty}^{\infty} \int_{-\infty}^{\infty} \int_{-\infty}^{\infty} f^{3 \mathrm{D}}(\mathbf{v}) \delta(\mathbf{v} \cdot \hat{\mathbf{u}}-u) d \mathbf{v},
$$

where $g(u)$ is the projected velocity distribution function, $u$ is the velocity projected onto the unit vector $\hat{\mathbf{u}}$ in the projection direction, and $\delta$ is the Dirac $\delta$-function. For axisymmetric $3 \mathrm{D}$ functions in velocity space, the integration over the ignorable gyroangle can be calculated analytically using so-called weight functions. ${ }^{18}$ In $\left(v_{\|}, v_{\perp}\right)$-space and in $(\mathrm{E}, \mathrm{p})$-space, Eq. (32) becomes, ${ }^{19}$ respectively,

$$
\begin{aligned}
& g(u, \phi)=\int_{0}^{\infty} \int_{-\infty}^{\infty} w_{\mathrm{Car}}\left(v_{\|}, v_{\perp}, u, \phi\right) f_{\mathrm{Car}}^{2 \mathrm{D}}\left(v_{\|}, v_{\perp}\right) d v_{\|} d v_{\perp} \text { and } \\
& g(u, \phi)=\int_{0}^{\infty} \int_{-1}^{1} w_{\mathrm{E} p}(\mathrm{E}, p, u, \phi) f_{\mathrm{E} p}^{2 \mathrm{D}}(\mathrm{E}, p) d p d \mathrm{E},
\end{aligned}
$$

where $\phi$ is the angle between the unit vector in projection direction $\hat{\mathbf{u}}$ and the magnetic field. One angle is sufficient to describe the projection direction due to the axisymmetry. The weight function in $\left(v_{\|}, v_{\perp}\right)$-space and in $(E, p)$-space is, respectively, ${ }^{19}$

$$
\begin{aligned}
& w_{\mathrm{Car}}\left(v_{\|}, v_{\perp}, u, \phi\right)=\frac{1}{\pi v_{\perp} \sin \phi \sqrt{1-\left(\frac{u-v_{\|} \cos \phi}{v_{\perp} \sin \phi}\right)^{2}}} \text { and } \\
& w_{\mathrm{Ep}}(\mathrm{E}, p, u, \phi) \\
& =\frac{1}{\pi \sqrt{\left(1-p^{2}\right) 2 \mathrm{E} / m} \sin \phi \sqrt{1-\left(\frac{u / \sqrt{2 \mathrm{E} / m}-p \cos \phi}{\sqrt{1-p^{2}} \sin \phi}\right)^{2}}} .
\end{aligned}
$$

This completes the discussion of the most common velocity or energy distribution functions encountered in the fusion plasma physics literature. Finally, we specify the limits to obtain the full densities from each function, which provides us with a normalization condition that we will enforce for all distributions in the following. The density is in each case obtained by integration over velocity-space

$$
\begin{gathered}
n=\int_{-\infty}^{\infty} \int_{-\infty}^{\infty} \int_{-\infty}^{\infty} f_{\mathrm{Car}}^{3 \mathrm{D}}\left(v_{\|}, v_{\perp 1}, v_{\perp 2}\right) d v_{\|} d v_{\perp 1} d v_{\perp 2}, \\
n=\int_{-\infty}^{\infty} \int_{-\infty}^{\infty} \int_{-\infty}^{\infty} f_{\mathrm{C} a r}^{3 \mathrm{D}}\left(v_{\|}, v_{\perp}\right) d v_{\|} d v_{\perp 1} d v_{\perp 2}, \\
n=\int_{0}^{2 \pi} \int_{0}^{\infty} \int_{-\infty}^{\infty} f_{c y l}^{3 \mathrm{D}}\left(v_{\|}, v_{\perp}\right) d v_{\|} d v_{\perp} d \gamma \\
n=\int_{0}^{\infty} \int_{-\infty}^{\infty} f_{\mathrm{Car}}^{2 \mathrm{D}}\left(v_{\perp}, v_{\perp}\right) d v_{\|} d v_{\perp} \\
n=\int_{-1}^{1} \int_{0}^{\infty} f_{\mathrm{Ep}}^{2 \mathrm{D}}(\mathrm{E}, p) d \mathrm{E} d p \\
n=\int_{-1}^{1} \int_{0}^{\infty} f_{v p}^{2 \mathrm{D}}(v, p) d v d p \\
n=\int_{0}^{\infty} f_{v}^{1 \mathrm{D}}(v) d v \\
n=\int_{0}^{\infty} f_{\mathrm{E}}^{1 \mathrm{D}}(\mathrm{E}) d \mathrm{E} \\
n=\int_{-\infty}^{\infty} g^{1 \mathrm{D}}(u) d u
\end{gathered}
$$

Alternatively, one may enforce that the respective integral gives unity to obtain a probability density function.

\section{MAXWELLIAN IN VARIOUS COORDINATE SYSTEMS}

We first illustrate the transformation of velocity distribution functions to the coordinate systems discussed in Sec. II for an ordinary Maxwellian, which is perhaps the most widely 
known distribution function in physics and which is therefore well-suited as a first practical example. It can be written as

$$
f^{3 \mathrm{D}}(\mathbf{v})=\frac{n}{\pi^{3 / 2} v_{t}^{3}} \exp \left(-\frac{\mathbf{v}^{2}}{v_{t}^{2}}\right),
$$

where $v_{t}$ is the thermal speed

$$
v_{t}=\sqrt{2 \mathrm{~T} / m} .
$$

$\mathrm{T}$ is the temperature in units of energy. The conversion from Kelvin to Joules is done using Boltzmann's constant $k=1.38 \times 10^{-23} \mathrm{~J} / \mathrm{K}$ and from electronvolt to Joules using $1.6 \times 10^{-19} \mathrm{~J} / \mathrm{eV}$. The prefactor of the exponential ensures that the integral over 3D velocity space gives the density. The Maxwellian can be written in 3D Cartesian coordinates as

$$
f_{\mathrm{Car}}^{3 \mathrm{D}}\left(v_{\|}, v_{\perp 1}, v_{\perp 2}\right)=\frac{n}{\pi^{3 / 2} v_{t}^{3}} \exp \left(-\frac{v_{\|}^{2}+v_{\perp 1}^{2}+v_{\perp 2}^{2}}{v_{t}^{2}}\right) .
$$

A slice of this function with $v_{\perp 2}=0$ is illustrated in Fig. 1(a). The parameters are set to model a typical plasma in ITER. The density is obtained from the triple integral in Eq. (35) which is easily solved since it can be separated into the product of three identical integrals of the form

$$
\int_{-\infty}^{\infty} \exp \left(-\frac{v_{x}^{2}}{v_{t}^{2}}\right) d v_{x}=\sqrt{\pi} v_{t}
$$

so that Eq. (35) is obeyed.

Due to the axisymmetry in magnetized plasmas, we can describe the Maxwellian in $\left(v_{\|}, v_{\perp}\right)$-coordinates

$$
f_{\mathrm{Car}}^{3 \mathrm{D}}\left(v_{\|}, v_{\perp}\right)=\frac{n}{\pi^{3 / 2} v_{t}^{3}} \exp \left(-\frac{v_{\|}^{2}+v_{\perp}^{2}}{v_{t}^{2}}\right) .
$$

As mentioned in Sec. II, this representation refers to a 3D Cartesian velocity space density parametrized in cylindrical coordinates. The Maxwellian in $\left(v_{\|}, v_{\perp}\right)$ coordinates is illustrated in Fig. 1(b). Note that $\left(v_{\|}, v_{\perp 1}, v_{\perp 2}\right) \in(-\infty ; \infty)$, whereas $v_{\perp} \in[0 ; \infty)$. The Maxwellian fully transformed to 3D cylindrical coordinates is

$$
f_{\text {cyl }}^{3 \mathrm{D}}\left(v_{\|}, v_{\perp}\right)=\frac{n v_{\perp}}{\pi^{3 / 2} v_{t}^{3}} \exp \left(-\frac{v_{\|}^{2}+v_{\perp}^{2}}{v_{t}^{2}}\right) .
$$

Equations (48) and (49) differ by $v_{\perp}$ which is the Jacobian of the transformation from Cartesian to cylindrical coordinates. However, Eq. (49) is not often used in this form, but it is integrated over the gyroangle $\gamma$ to obtain the Maxwellian in a 2D Cartesian coordinate system as $f_{\text {Car }}^{2 \mathrm{D}}\left(v_{\|}, v_{\perp}\right)$ with $v_{\perp}>0$ and no implied third direction

$$
f_{\mathrm{C} a r}^{2 \mathrm{D}}\left(v_{\|}, v_{\perp}\right)=\frac{2 n v_{\perp}}{\sqrt{\pi} v_{t}^{3}} \exp \left(-\frac{v_{\|}^{2}+v_{\perp}^{2}}{v_{t}^{2}}\right) .
$$

The Maxwellian in this 2D Cartesian coordinate system is illustrated in Fig. 1(c). The isotropy in velocity space is not apparent in $2 \mathrm{D}\left(v_{\|}, v_{\perp}\right)$-coordinates due to the Jacobian $v_{\perp}$

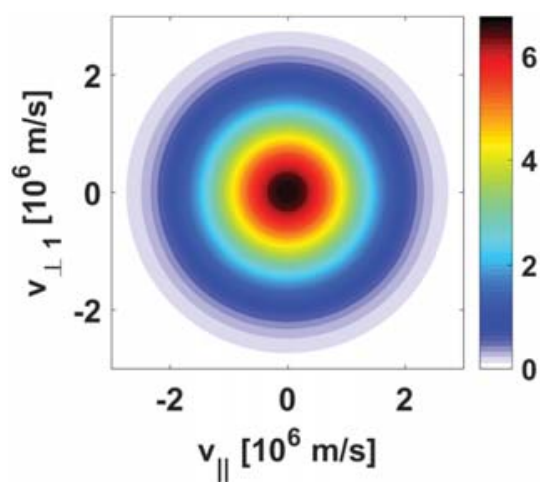

(a) $f_{C a r}^{3 D}\left(v_{\|}, v_{\perp 1}, v_{\perp 2}\right)$

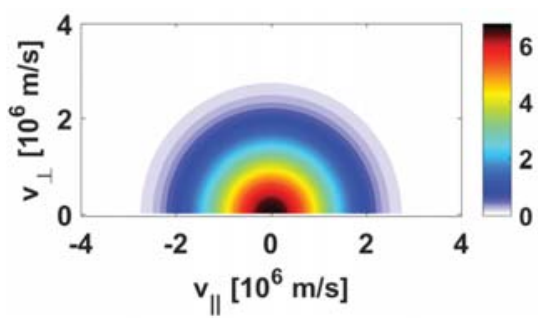

(b) $f_{\text {Car }}^{3 D}\left(v_{\|}, v_{\perp}\right)$

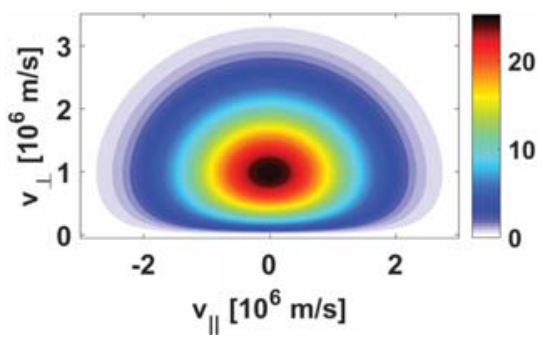

(c) $f_{\text {Car }}^{2 D}\left(v_{\|}, v_{\perp}\right)$

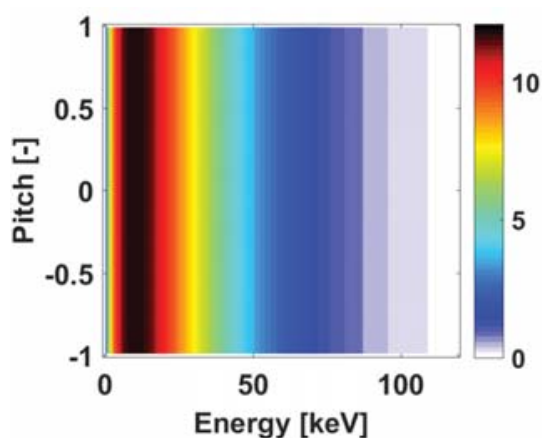

(d) $f_{E p}^{2 D}(E)$

FIG. 1. Maxwellian with ITER-relevant parameters $T=20 \mathrm{keV}$ and $n=10^{20} \mathrm{~m}^{-3}$ in various coordinate systems. (a) $f_{\mathrm{Car}}^{3 D}\left(v_{\|}, v_{\perp 1}, v_{\perp 2}\right)$. (b) $f_{\mathrm{Car}}^{3 D}\left(v_{\|}, v_{\perp}\right)$. (c) $f_{\mathrm{Car}}^{2 D}$ $\left(v_{\|}, v_{\perp}\right)$. (d) $f_{E p}^{2 D}(E)$. The units in (a) and (b) are $\mathrm{s}^{3} / \mathrm{m}^{6}$, in (c) $10^{6} \mathrm{~s}^{2} / \mathrm{m}^{5}$, and in (d) $10^{14} /\left(\mathrm{eV} \mathrm{m}^{3}\right)$. The isotropy of the Maxwellian is reflected in the concentric isolevels in (a) and (b) and in the dependence of the function on the pitch in (d). However, in (c), the Jacobian $v_{\perp}$ conceals the isotropy as $f_{\text {Car }}^{2 D}$ approaches zero close to the $v_{\|}$axis, where $v_{\perp} \rightarrow 0$. Likewise, $f_{E p}^{2 D}(E)$ approaches zero for $E \rightarrow 0$. 
which is presumably why the representation in Fig. 1(b) is often preferred.

The Maxwellian in 2D (E, $p)$-space is after transformation according to Eqs. (14) and (15)

$$
f_{\mathrm{Ep}}^{2 \mathrm{D}}(\mathrm{E})=n \sqrt{\frac{\mathrm{E}}{\pi \mathrm{T}^{3}}} \exp \left(-\frac{E}{\mathrm{~T}}\right),
$$

where the ignorable pitch does not appear due to isotropy. The Maxwellian in (E, p)-coordinates is illustrated in Fig. 1(d).

The Maxwellian in $(v, p)$-coordinates is

$$
f_{v p}^{2 \mathrm{D}}(v)=\frac{2 n v^{2}}{\sqrt{\pi} v_{t}^{3}} \exp \left(-\frac{v^{2}}{v_{t}^{2}}\right),
$$

where again $p$ does not appear.

Next, we turn to 1D descriptions in terms of the speed, the energy, and the projected velocity. These are useful in many physical situations other than magnetized plasma and are therefore often covered in textbooks of basic and statistical physics. The Maxwellian in 3D Cartesian velocity space parametrized in the spherical coordinates $(v, \eta, \zeta)$ is

$$
f_{\text {Car }}^{3 \mathrm{D}}(v)=\frac{n}{\pi^{3 / 2} v_{t}^{3}} \exp \left(-\frac{v^{2}}{v_{t}^{2}}\right) .
$$

The Maxwellian fully transformed to 3D spherical coordinates is

$$
f_{\text {sph }}^{3 \mathrm{D}}(v, \zeta)=\frac{n v^{2} \sin \zeta}{\pi^{3 / 2} v_{\mathrm{t}}^{3}} \exp \left(-\frac{v^{2}}{v_{\mathrm{t}}^{2}}\right) .
$$

Integration over $\eta$ and $\zeta$ but not over $v$ immediately gives the $1 \mathrm{D}$ distribution of speeds

$$
f_{v}^{1 \mathrm{D}}(v)=\frac{4 n v^{2}}{\sqrt{\pi} v_{\mathrm{t}}^{3}} \exp \left(-\frac{v^{2}}{v_{t}^{2}}\right)
$$

in units of $\mathrm{s} / \mathrm{m}^{4}$. The energy distribution or energy spectrum for the Maxwellian is obtained from Eq. (55) by coordinate transformation according to Eq. (22)

$$
f_{\mathrm{E}}^{1 \mathrm{D}}(\mathrm{E})=2 n \sqrt{\frac{\mathrm{E}}{\pi \mathrm{T}^{3}}} \exp \left(-\frac{E}{\mathrm{~T}}\right) .
$$

Equations (55) and (56) can also be derived from Eqs. (52) and (51) by integration over the pitch, respectively, which gives a factor two since the Maxwellian is isotropic and does not depend on the pitch

$$
\begin{gathered}
f_{\mathrm{E}}^{\mathrm{D}}(\mathrm{E})=\int_{-1}^{1} f_{\mathrm{E} p}^{2 \mathrm{D}}(\mathrm{E}, p) d p=2 f_{\mathrm{E} p}^{2 \mathrm{D}}(\mathrm{E}), \\
f_{v}^{1 \mathrm{D}}(v)=\int_{-1}^{1} f_{\mathrm{E} p}^{2 \mathrm{D}}(v, p) d p=2 f_{v p}^{2 \mathrm{D}}(v) .
\end{gathered}
$$

Equations (57) and (58) hold for any isotropic velocity distribution function.

Finally, the $1 \mathrm{D}$ projection of the Maxwellian is highly important for the many diagnostics relying on the Doppler shift. As the Maxwellian is isotropic, the projection is the same in any arbitrary direction described by the unit vector $\hat{\mathbf{u}}$. As the three perpendicular coordinates are separable, we can hence compute the projected velocity distribution function onto any coordinate axis by effecting only two of the three integrals

$$
g(u)=\frac{n}{\sqrt{\pi} v_{t}} \exp \left(-\frac{u^{2}}{v_{t}^{2}}\right) .
$$

The 1D distribution of projected velocities $u$ has the form of a 1D Maxwellian for any direction. We note that $u \in(-\infty ; \infty)$, whereas $v, E \in[0 ; \infty)$.

This completes the transformation of the ordinary Maxwellian to the most common velocity space and energy space coordinate systems in fusion plasma physics and the diagnostically important projection onto a particular direction. Section IV gives these equations for a drifting bi-Maxwellian.

\section{DRIFTING BI-MAXWELLIAN}

In magnetized fusion plasmas, the ions are usually not in thermal equilibrium, but they are anisotropic to some degree. One way to allow departure from thermal equilibrium is to allow different temperatures in parallel and perpendicular directions with respect to the magnetic field, for example, as a consequence of anisotropic plasma heating schemes. ${ }^{20}$ ICRF couples to the gyration of the ions which occurs in the perpendicular direction. This increases the velocity components perpendicular to the magnetic field. NBI can also heat preferentially in parallel or perpendicular directions. In such cases, we can think of a parallel temperature $T_{\|}$and a perpendicular temperature $T_{\perp}$ in a bi-Maxwellian velocity distribution function. ${ }^{13,21-25}$ The very large perpendicular temperature obtained by ICRF heating is sometimes called the tail temperature. The plasma may also drift as a whole relative to the coordinate system. Since drift as a whole does not change the equilibrium, it can be introduced in a Galilean transformation. ${ }^{2}$ We start by considering the completely arbitrarily drifting "tri-Maxwellian" with three temperatures in 3D Cartesian coordinates

$$
\begin{aligned}
f_{\text {Car }}^{3 \mathrm{D}}\left(v_{\|}, v_{\perp 1}, v_{\perp 2}\right)= & \frac{n}{\pi^{3 / 2} v_{t, \|} v_{t, \perp 1} v_{t, \perp 2}} \times \exp \left(-\frac{\left(v_{\|}-v_{d \|}\right)^{2}}{v_{t, \|}^{2}}\right. \\
& \left.-\frac{\left(v_{\perp 1}-v_{d \perp 1}\right)^{2}}{v_{t, \perp 1}^{2}}-\frac{\left(v_{\perp 2}-v_{d \perp 2}\right)^{2}}{v_{t, \perp 2}^{2}}\right),
\end{aligned}
$$

where

$$
\begin{aligned}
v_{t, \|} & =\sqrt{2 \mathrm{~T}_{\|} / m}, \\
v_{t, \perp 1} & =\sqrt{2 \mathrm{~T}_{\perp 1} / m},
\end{aligned}
$$

and

$$
v_{\mathrm{t}, \perp 2}=\sqrt{2 \mathrm{~T}_{\perp 2} / m} .
$$

Equation (60) is not typical for a magnetized plasma since the temperatures in the two perpendicular directions are the same due to the fast gyration. The basic assumption of the bi- 
Maxwellian is hence that $T_{\perp 1}=T_{\perp 2}=T_{\perp}$, whereas $T_{\|}$is allowed to be different. The corresponding thermal velocity is defined as

$$
v_{\mathrm{t}, \perp}=\sqrt{2 \mathrm{~T}_{\perp} / m}
$$

and hence, $v_{t, \perp 1}=v_{t, \perp 2}=v_{t, \perp}$. Further, one may align one of the perpendicular coordinate directions with the perpendicular drift velocity component without the loss of generality, for example, $v_{d \perp 1}=v_{d \perp}$ and $v_{d \perp 2}=0$

$$
\begin{aligned}
f_{\mathrm{Car}}^{3 \mathrm{D}}\left(v_{\|}, v_{\perp 1}, v_{\perp 2}\right)= & \frac{n}{\pi^{3 / 2} v_{\mathrm{t}, \|} v_{\mathrm{t}, \perp}^{2}} \times \exp \left(-\frac{\left(v_{\|}-v_{d \|}\right)^{2}}{v_{\mathrm{t}, \|}^{2}}\right. \\
& \left.-\frac{\left(v_{\perp 1}-v_{d \perp}\right)^{2}+v_{\perp 2}^{2}}{v_{\mathrm{t}, \perp}^{2}}\right) .
\end{aligned}
$$

Substitution of cylindrical coordinates according to Eq. (8) gives

$$
\begin{aligned}
f_{\mathrm{Car}}^{3 \mathrm{D}}\left(v_{\|}, v_{\perp}, \gamma\right)= & \frac{n}{\pi^{3 / 2} v_{t, \|} v_{t, \perp}^{2}} \times \exp \left(-\frac{\left(v_{\|}-v_{d \|}\right)^{2}}{v_{t, \|}^{2}}\right. \\
& \left.-\frac{v_{\perp}^{2}-2 v_{\perp} v_{d \perp} \cos \gamma+v_{d \perp}^{2}}{v_{t, \perp}^{2}}\right) .
\end{aligned}
$$

In either coordinates, the arbitrarily drifting bi-Maxwellian is described by three coordinates. The gyro-angle $\gamma$ appears in Eq. (66) because any perpendicular drift, which is common in magnetized plasma, breaks the axisymmetry in the lab frame. The axisymmetry can be restored by a coordinate transformation to the guiding center frame in the perpendicular direction such that $v_{d \perp}=0$.

Parallel drift does not break the axisymmetry. The purely parallel-drifting bi-Maxwellian is also a useful model in many plasma scenarios since drift in the parallel direction is often much larger than the drift in the perpendicular direction in the lab frame. For $v_{d \perp}=0$, the gyroangle $\gamma$ drops out and we obtain the axisymmetric (about B) parallel-drifting bi-Maxwellian parameterized in cylindrical coordinates

$$
f_{\mathrm{C} a r}^{3 \mathrm{D}}\left(v_{\|}, v_{\perp}\right)=\frac{n}{\pi^{3 / 2} v_{t, \|} v_{t, \perp}^{2}} \exp \left(-\frac{\left(v_{\|}-v_{d \|}\right)^{2}}{v_{\mathrm{t}, \|}^{2}}-\frac{v_{\perp}^{2}}{v_{t, \perp}^{2}}\right) .
$$

In the $3 \mathrm{D}$ cylindrical coordinate representation including the Jacobian, we get

$$
f_{c y l}^{3 \mathrm{D}}\left(v_{\|}, v_{\perp}\right)=\frac{n v_{\perp}}{\pi^{3 / 2} v_{t, \|} v_{t, \perp}^{2}} \exp \left(-\frac{\left(v_{\|}-v_{d \|}\right)^{2}}{v_{t, \|}^{2}}-\frac{v_{\perp}^{2}}{v_{t, \perp}^{2}}\right) .
$$

This representation is often not as useful as the 3D Cartesian phase-space density where it is easier to spot any anisotropy in the parallel and perpendicular temperatures in a graph. In 2D Cartesian $\left(v_{\|}, v_{\perp}\right)$-space, the parallel-drifting bi-Maxwellian is

$$
f_{\mathrm{Car}}^{2 \mathrm{D}}\left(v_{\|}, v_{\perp}\right)=\frac{2 n v_{\perp}}{\sqrt{\pi} v_{\mathrm{t}, \|} v_{t, \perp}^{2}} \exp \left(-\frac{\left(v_{\|}-v_{d \|}\right)^{2}}{v_{t, \|}^{2}}-\frac{v_{\perp}^{2}}{v_{\mathrm{t}, \perp}^{2}}\right),
$$

and in 2D (E, p)-space, according to Eqs. (14) and (15)
$f(E, p)=n\left(\frac{E}{\pi T_{\perp}^{2} T_{\|}}\right)^{1 / 2} \times \exp \left(-\frac{\left(p \sqrt{E}-\sqrt{\frac{m}{2}} v_{d \|}\right)^{2}}{T_{\|}}-\frac{\left(1-p^{2}\right) E}{T_{\perp}}\right)$.

The isotropic Maxwellians from Sec. III can be recovered by setting $\mathrm{T}=\mathrm{T}_{\|}=\mathrm{T}_{\perp}$ and $v_{d \|}=0$. The effects of drift and temperature anisotropy for the drifting bi-Maxwellian are shown in the various coordinate systems in Fig. 2 as compared to the nominal ITER scenario from Fig. 1.

It is straightforward to integrate Eq. (70) over the pitch to obtain the energy spectrum of the bi-Maxwellian. But since the bi-Maxwellian is a 2D function, the energy spectrum and the speed distribution do not appear to be directly useful. However, the 1D projection of the bi-Maxwellian is important to understand diagnostics relying on the Doppler shift. ${ }^{25}$ The $1 \mathrm{D}$ projection of the isotropic Maxwellian was given in Sec. III based on the isotropy of the function. The $1 \mathrm{D}$ projection of the anisotropic bi-Maxwellian unsurprisingly does depend on the direction and is hence not as straightforward to compute. Nevertheless, the integrals in Eq. (33) can be solved analytically for axisymmetric bi-Maxwellians, i.e., bi-Maxwellians without perpendicular drift. $^{25}$ Any perpendicular drift can be handled by Galilean transformations. After the Galilean transformations and integration, we find that an arbitrarily drifting bi-Maxwellian has an intuitive $1 \mathrm{D}$ projection $^{25}$

$$
g(u)=n\left(\frac{m}{2 \pi \mathrm{T}_{u}}\right)^{1 / 2} \exp \left(-\frac{m\left(u-u_{d}\right)^{2}}{2 \mathrm{~T}_{u}}\right)
$$

where we have introduced an effective temperature in the $u$ coordinate along the line-of-sight

$$
\mathrm{T}_{u}=\mathrm{T}_{\perp} \sin ^{2} \phi+\mathrm{T}_{\|} \cos ^{2} \phi
$$

and an effective $u$-drift

$$
u_{d}=v_{d \|} \cos \phi+v_{d \perp} \cos \beta,
$$

where $\beta$ is the angle between the perpendicular drift and the line-of-sight. Equation (71) thus has the form of a drifting 1D Maxwellian in the projected velocity $u$. Equation (71) relates the drifting 1D Maxwellian often used in temperature and drift velocity (so-called "rotation") measurements to an underlying group of 2D arbitrarily drifting bi-Maxwellians with the same 1D projection. This ambiguity can only be resolved by additional information, for example, an additional measurement. 1D projections of a drifting bi-Maxwellian along various directions are compared in Fig. 3. The anisotropic bi-Maxwellian has a symmetric projection perpendicular to the magnetic field. The effect of parallel drift is largest for $\phi=0^{\circ}$.

\section{RING DISTRIBUTION}

In magnetic fusion plasmas, it is often undesirable that the velocity distribution function has a local maximum at large velocities, a situation termed the bump-on-tail. A bump-on-tail is a source of free energy that can drive plasma instabilities. Such a bump-on-tail may be described by a ring distribution 


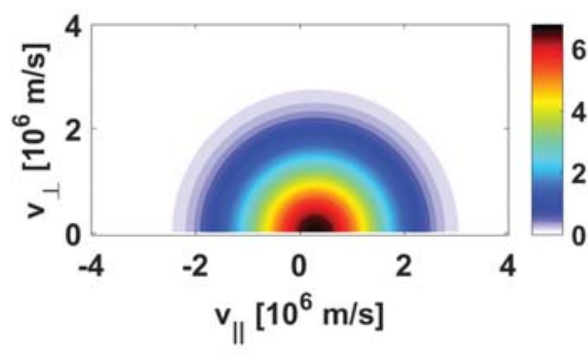

(a) $f_{C a r}^{3 D}\left(v_{\|}, v_{\perp}\right)$

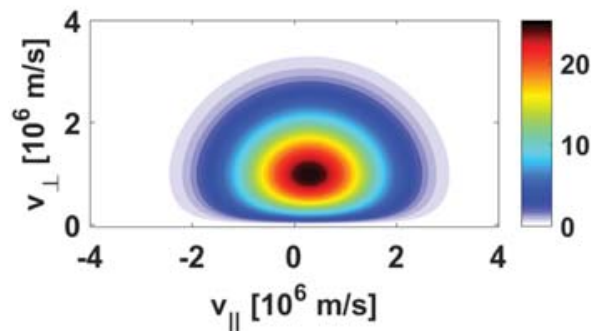

(c) $f_{C a r}^{2 D}\left(v_{\|}, v_{\perp}\right)$

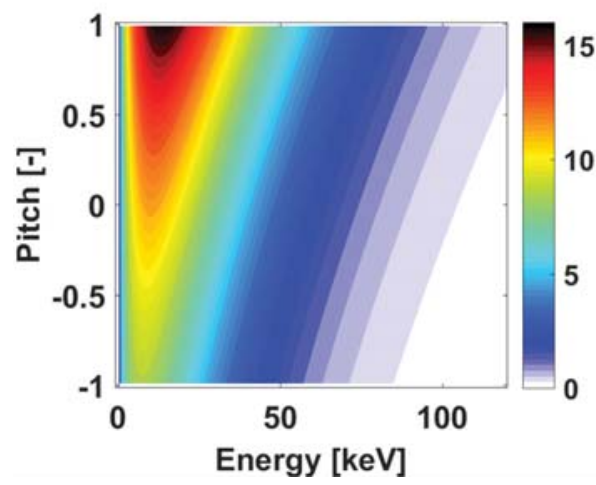

(e) $f_{E p}^{2 D}(E, p)$

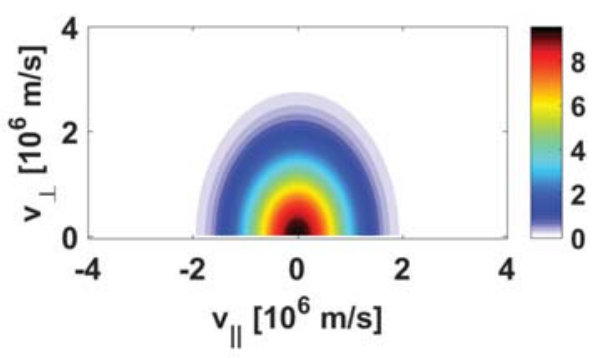

(b) $f_{C a r}^{3 D}\left(v_{\|}, v_{\perp}\right)$

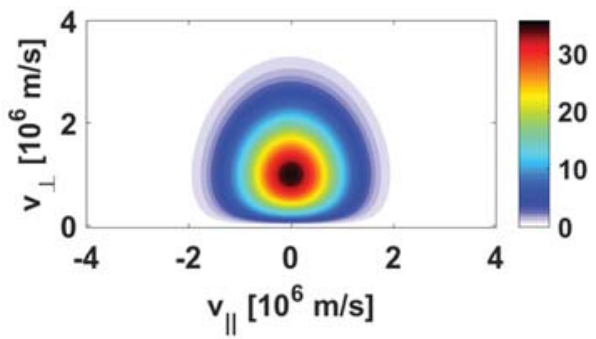

(d) $f_{C a r}^{2 D}\left(v_{\|}, v_{\perp}\right)$

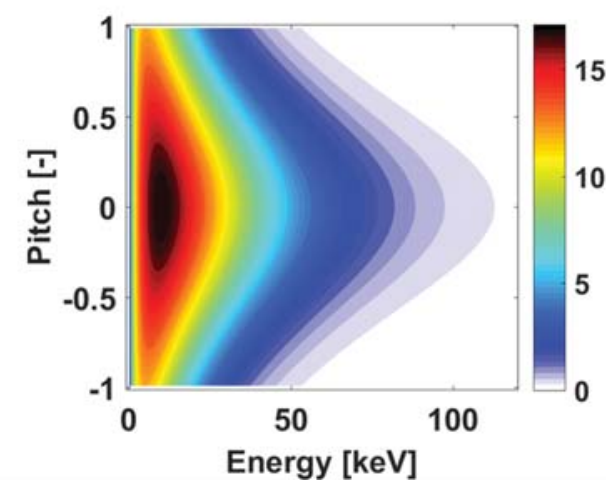

(f) $f_{E p}^{2 D}(E, p)$
FIG. 2. Drifting Maxwellians and biMaxwellians in three coordinate systems. The baseline parameters are $T=20 \mathrm{keV}$ and $n=10^{20} \mathrm{~m}^{-3}$ as in Fig. 1. However, in (a), (c), and (e), we set $v_{d}=3 \times 10^{5} \mathrm{~m}$ $\mathrm{s}$, and in (b), (d), and (f), we set $T_{\|}$ $=10 \mathrm{keV}$ and $T_{\perp}=20 \mathrm{keV}$. Drift is reflected in the translation in the $v_{\|}$-direction for $\left(v_{\|}, v_{\perp}\right)$-coordinates and in the bias towards positive $p$ in $(E, p)$-coordinates. Temperature anisotropy is reflected in the departure from the circular shape in (b) and from straight lines in (f).

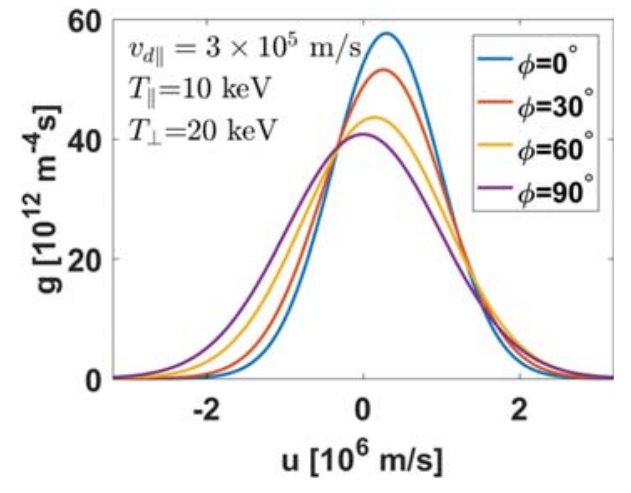

FIG. 3. 1D projections at various projection angles of a bi-Maxwellian with drift parallel to the magnetic field. The more parallel the view, the narrower the function due to the smaller temperature and the larger the shift in $u$ due to the parallel drift velocity. which is sometimes done in stability calculations. ${ }^{26,27}$ A Gaussian ring distribution in the various coordinate systems is illustrated in Fig. 4. A ring distribution can also be used as the model for fast ions immediately after an NBI is switched on. Right after birth, the NBI ions have the velocity of the beam, but a ring distribution is formed quickly in 3D velocity space due to the gyration of the ions. Finally, the ring distribution can serve as a basic building block for arbitrary velocity distribution functions which can be constructed by adding ring distributions with various velocities. $^{14,19,28}$

As an idealized model, we can first consider the cold ring distribution. ${ }^{29,30}$ In this case, the Gaussian in the zero-width limit is replaced by the Dirac $\delta$-function. The cold ring can be represented as a single point in $2 \mathrm{D}$ velocity space. In $3 \mathrm{D}$ Cartesian, 3D cylindrical, 2D Cartesian, and (E, $p$ )-coordinates, the cold ring distribution is

$$
f_{\text {Car }}^{3 \mathrm{D}}\left(v_{\|}, v_{\perp}\right)=\frac{n}{2 \pi v_{\perp}} \delta\left(v_{\|}-v_{0 \|}\right) \delta\left(v_{\perp}-v_{0 \perp}\right)
$$




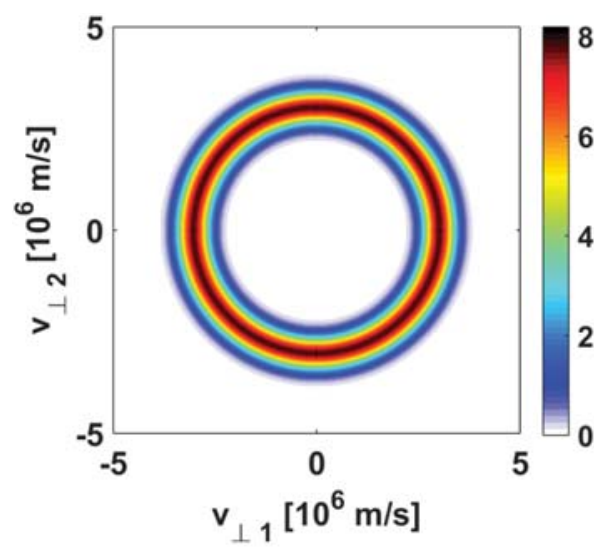

(a) $f_{C a r}^{3 D}\left(v_{\|}, v_{\perp 1}, v_{\perp 2}\right)$

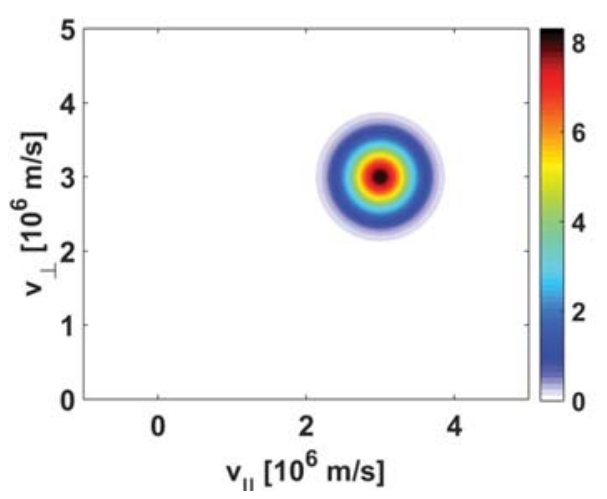

(c) $f_{\text {Car }}^{2 D}\left(v_{\|}, v_{\perp}\right)$

$f_{\text {cyl }}^{3 \mathrm{D}}\left(v_{\|}, v_{\perp}, \gamma\right)=\frac{n}{2 \pi} \delta\left(v_{\|}-v_{0 \|}\right) \delta\left(v_{\perp}-v_{0 \perp}\right)$,

$f_{\mathrm{Car}}^{2 \mathrm{D}}\left(v_{\|}, v_{\perp}\right)=n \delta\left(v_{\|}-v_{0 \|}\right) \delta\left(v_{\perp}-v_{0 \perp}\right)$,

$f_{\mathrm{Ep}}^{2 \mathrm{D}}(\mathrm{E}, p)=n \delta\left(\mathrm{E}-\mathrm{E}_{0}\right) \delta\left(p-p_{0}\right)$.

The energy spectrum and the 1D speed distribution are obtained by integrating Eq. (77) over the pitch and by transforming to the speed

$$
\begin{gathered}
f_{\mathrm{E}}^{1 \mathrm{D}}(\mathrm{E})=n \delta\left(\mathrm{E}-\mathrm{E}_{0}\right), \\
f_{v}^{1 \mathrm{D}}(v)=n \delta\left(v-v_{0}\right) .
\end{gathered}
$$

The pitch information is lost in these functions, such that these $1 \mathrm{D}$ representations not necessarily represent ring distributions, but other 2D functions can have the same energy and speed spectra.

The 1D projection of the cold ring distribution is of great interest for diagnostics relying on the Doppler shift because it shows the footprint of an ion at a particular point in velocity space in the measurable spectrum. ${ }^{19,28}$ Substitution of Eq. (76) into Eq. (33) and integration give

$$
g(u)=\frac{n}{\pi v_{0 \perp} \sin \phi \sqrt{1-\left(\frac{u-v_{0 \|} \cos \phi}{v_{0 \perp} \sin \phi}\right)^{2}}} .
$$

Equation (80) is proportional (by a factor $n$ ) to the probability density function in $u$ of the projection equation $^{19}$

$$
u=v_{0 \|} \cos \phi+v_{0 \perp} \sin \phi \cos \gamma
$$

The distribution function is centered at $v_{0 \|} \cos \phi$ and has a width of $2 v_{0 \perp} \sin \phi$ since $\cos \gamma \in[-1 ; 1]$. An example of the projected ring distribution is shown in Fig. 5.

A warm ring distribution is obtained by replacing the Dirac $\delta$-function by the normal distribution. ${ }^{31-33}$ The scaling follows from the requirement that the integral over velocity space gives the density. We obtain in 2D Cartesian coordinates

$$
\begin{aligned}
f_{\text {Car }}^{2 \mathrm{D}}\left(v_{\|}, v_{\perp}\right)= & \frac{2 n}{\pi w_{\|} w_{\perp}\left(\operatorname{erf}\left(\frac{v_{0 \perp}}{w_{\perp}}\right)+1\right)} \\
& \times \exp \left(-\frac{\left(v_{\|}-v_{0 \|}\right)^{2}}{w_{\|}^{2}}-\frac{\left(v_{\perp}-v_{0 \perp}\right)^{2}}{w_{\perp}^{2}}\right),
\end{aligned}
$$

and in 3D Cartesian velocity space parametrized in cylindrical coordinates 


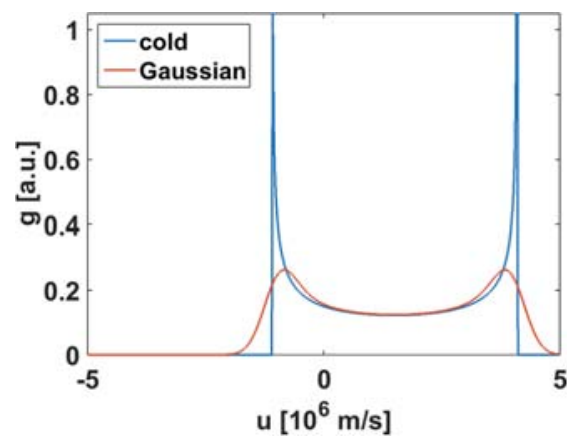

FIG. 5. 1D projections of a cold ring distribution and a warm ring distribution where the $\delta$-function is replaced by a Gaussian function in $2 \mathrm{D}\left(v_{\|}, v_{\perp}\right)$-space. The center of the function is at $v_{\| 0} \cos \phi$, and the width is $v_{\perp 0} \sin \phi$.

$$
\begin{aligned}
f_{\text {Car }}^{3 \mathrm{D}}\left(v_{\|}, v_{\perp}\right)= & \frac{n}{\pi^{2} w_{\|} w_{\perp} v_{\perp}\left(\operatorname{erf}\left(\frac{v_{0 \perp}}{w_{\perp}}\right)+1\right)} \\
& \times \exp \left(-\frac{\left(v_{\|}-v_{0 \|}\right)^{2}}{w_{\|}^{2}}-\frac{\left(v_{\perp}-v_{0 \perp}\right)^{2}}{w_{\perp}^{2}}\right),
\end{aligned}
$$

where $w_{\|}$and $w_{\perp}$ are the Gaussian widths of the ring in parallel and perpendicular directions, respectively. Here, the error function appears because the normal distribution is restricted to $v_{\perp}$ $>0$. For $v_{0 \perp} \gg w_{\perp}$, the scaling factor in Eq. (82) approaches $n /\left(\pi w_{\|} w_{\perp}\right)$ corresponding to the scaling for normal distributions that are not cut off. Finally, we transform the ring distribution to $(\mathrm{E}, p)$-coordinates

$$
\begin{aligned}
f_{\mathrm{Ep}}^{2 \mathrm{D}}(\mathrm{E}, p)= & \frac{2 n}{\pi w_{\|} w_{\perp}\left(\operatorname{erf}\left(\frac{v_{0 \perp}}{w_{\perp}}\right)+1\right)} \frac{1}{m \sqrt{1-p^{2}}} \\
& \times \exp \left(-\frac{\left(p \sqrt{2 \mathrm{E} / m}-v_{0 \|}\right)^{2}}{w_{\|}^{2}}-\frac{\left(\sqrt{\left(1-p^{2}\right) 2 \mathrm{E} / m}-v_{0 \perp}\right)^{2}}{w_{\perp}^{2}}\right) .
\end{aligned}
$$

A closed form analytical expression for the projection of a warm ring distribution would be useful but is not yet available. It is therefore necessary to calculate such projections numerically which is possible by a formalism for arbitrary distribution functions that are axisymmetric about $\mathbf{B} .{ }^{34}$ Projections of cold and warm ring distributions have double-humped shapes (Fig. 5).

\section{ISOTROPIC AND ANISOTROPIC SLOWING-DOWN DISTRIBUTIONS}

This section discusses isotropic and anisotropic slowingdown distributions. A fusion reactor has to balance the heat loss from the plasma by heat release from fusion reactions to maintain a steady-state plasma. The fusion energy is coupled to the plasma via $\alpha$-particles born at $3.5 \mathrm{MeV}$ in the $\mathrm{D}(\mathrm{T}, \mathrm{n}) \alpha$ fusion reaction. The $\alpha$-particles transfer their energy by collisions to the less energetic particles in the plasma which have an average energy about two orders of magnitude lower. The isotropic slowing-down distribution describes the classical slowing down of isotropically distributed high-energy ions due to collisions with electrons and ions in a thermal background plasma. ${ }^{8-10}$ It is a good model for the velocity distribution of $\alpha$-particles born in fusion reactions as the birth distribution can be assumed to be isotropic to a fairly good approximation. ${ }^{35}$ (However, the directional bias of NBI ions entering the DT reaction and the drift orbit topologies introduce some anisotropy, as do potentially Alfvén eigenmodes. ${ }^{36}$ )

The injection of neutral particle beams is perhaps the most widespread energetic particle source nowadays. Whereas the $\alpha$-particles from fusion reactions have roughly isotropically distributed birth velocities, the fast ions born by ionization of neutrals from NBI have a small pitch range given by the geometry of the NBI with respect to the magnetic field. This anisotropic source of ions consequently generates an anisotropic velocity distribution function. The injection energies are about $10 \mathrm{keV}-1 \mathrm{MeV}$. The ions are born at the nominal injection energy and, for positive-ion sources, at one-half and one-third of the full energy due to the acceleration of molecules with a single charge, e.g., $\mathrm{D}_{2}^{+}$and $\mathrm{D}_{3}^{+}$in a deuterium beam. Such distributions are fairly well described by anisotropic slowing-down distributions.

The slowing-down process is analytically described by a Fokker-Planck equation with a few simplifying assumptions. Here, we convey the basic idea and the necessary assumptions to arrive at both distributions. We assume axisymmetry about the magnetic field vector, small fast-ion densities, and velocities in the range of

$$
v_{\mathrm{t}, i} \ll v_{f} \ll v_{\mathrm{t}, e},
$$

where $v_{t, i}$ and $v_{t, e}$ are the thermal ion and electron speeds, respectively,

$$
v_{t, i}=\sqrt{\frac{2 \mathrm{~T}_{i}}{m_{i}}}, \quad v_{t, e}=\sqrt{\frac{2 \mathrm{~T}_{e}}{m_{e}}} .
$$

For these conditions, we can derive a homogeneous FokkerPlanck equation parametrized in $(v, p)$-coordinates with an anisotropic source term ${ }^{12}$

$$
\begin{aligned}
\frac{\partial f_{\mathrm{C} a r}^{3 \mathrm{D}}}{\partial \mathrm{t}}= & \frac{1}{\tau_{\mathrm{s}} v^{2}} \frac{\partial}{\partial v}\left(\left(v^{3}+v_{\mathrm{c}}^{3}\right) f_{\mathrm{C} a r}^{3 \mathrm{D}}\right)+\frac{\mathrm{Z}_{2} v_{\mathrm{c}}^{3}}{2 \tau_{\mathrm{s}} v^{3}} \frac{\partial}{\partial p}\left(\left(1-p^{2}\right) \frac{\partial f_{\mathrm{Car}}^{3 \mathrm{D}}}{\partial p}\right) \\
& +\frac{1}{\tau_{\mathrm{s}} v^{2}} \frac{\partial}{\partial v}\left(\frac{v^{2}}{m_{f}}\left(\mathrm{~T}_{e}+\frac{v_{\mathrm{c}}^{3}}{v^{3}} \mathrm{~T}_{i}\right) \frac{\partial f_{\mathrm{Car}}^{3 \mathrm{D}}}{\partial v}\right)+\mathrm{S}=0 .
\end{aligned}
$$

$\tau_{\mathrm{s}}$ is the Spitzer slowing-down time given by

$$
\tau_{\mathrm{s}}=\frac{3(2 \pi)^{3 / 2} \epsilon_{0}^{2} m_{f} \mathrm{~T}_{e}^{3 / 2}}{\mathrm{Z}_{f}^{2} e^{4} m_{e}^{1 / 2} n_{e} \ln \Lambda},
$$

where $\epsilon_{0}$ is the vacuum permittivity, $m_{f}$ is the fast-ion mass, $Z_{f}$ is the charge number of the fast ion, and $\ln \Lambda$ is the Coulomb logarithm. $v_{\mathrm{c}}$ is the crossover speed where drag on electrons equals drag on ions, given by

$$
v_{\mathrm{c}}=\left(\frac{3 \sqrt{\pi} m_{e}}{4 m_{f}} Z_{1}\right)^{1 / 3} v_{t, e}
$$


The effective charge numbers $Z_{1}$ and $Z_{2}$ are given by

$$
\begin{gathered}
Z_{1}=\sum_{i} \frac{n_{i} m_{f} Z_{i}^{2}}{n_{e} m_{i}} \\
Z_{2}=\sum_{i} \frac{n_{i} Z_{i}^{2}}{n_{e} Z_{1}} .
\end{gathered}
$$

For $n_{\mathrm{D}}=n_{\mathrm{T}}=n_{e} / 2$, we get $\mathrm{Z}_{1}=5 / 3 . \mathrm{S}$ is the source term which we will give separately for the isotropic and anisotropic cases.

As we seek a steady-state solution, we set the time derivative on the left-hand-side of Eq. (87) to zero. The first term on the right-hand-side is the only term with a single derivative which describes the frictional slowing down of the particles. The second and third terms have double derivatives and are hence diffusive in character (in velocity space): the second term is called pitch-angle scattering, and the third term is called speed diffusion. Speed diffusion is the only term leading to particle speeds larger than the birth speed and is consequently important for $v>v_{b}$. We focus on speeds below the birth speed $\left(v<v_{b}\right)$ where the speed diffusion term is small compared to the slowing-down term. ${ }^{12}$ Hence, we will neglect it in the following. We now solve this equation for isotropic and anisotropic source terms in Subsections VIA and VI B.

\section{A. Isotropic slowing-down distribution}

We start with the much simpler isotropic slowing-down distribution and seek a steady-state solution neglecting the small speed diffusion term. ${ }^{8-10,35}$ Further, we assume that the source term is isotropic and the ions are born at the birth speed such that

$$
\mathrm{S}(v)=\frac{\mathrm{S}_{0} \delta\left(v_{b}-v\right)}{4 \pi v^{2}} .
$$

For modelling an $\alpha$-particle distribution function, $\mathrm{S}_{0}$ is the fusion reaction rate

$$
\mathrm{S}_{0}=n_{\mathrm{D}} n_{\mathrm{T}}\langle\sigma v\rangle .
$$

The birth speed is $v_{b}=1.3 \times 10^{7} \mathrm{~m} / \mathrm{s}$ for $3.5 \mathrm{MeV} \alpha$-particles. The pitch angle diffusion term then vanishes due to the isotropic source term. The simplified Fokker-Planck equation becomes

$$
\frac{\partial}{\partial v}\left(\left(v^{3}+v_{\mathrm{c}}^{3}\right) f_{\mathrm{Car}}^{3 \mathrm{D}}(v)\right)+\frac{\mathrm{S}_{0} \tau_{\mathrm{s}} \delta\left(v-v_{b}\right)}{4 \pi}=0
$$

For $v \neq v_{b},\left(v^{3}+v_{\mathrm{c}}^{3}\right) f_{\mathrm{C} a r}^{3 \mathrm{D}}(v)$ must be a constant $\mathrm{C}$ as the $\delta$-function is zero. Further, for $v>v_{b}$, the distribution is zero as there is no acceleration and we have neglected speed diffusion. Hence, we can write the solution as

$$
f_{\text {Car }}^{3 \mathrm{D}}(v)=\mathrm{C} \frac{\mathrm{H}\left(v_{b}-v\right)}{v^{3}+v_{c}^{3}} .
$$

Substitution of Eq. (95) into Eq. (94) and integration over a small range around the birth speed give

$$
\mathrm{C}=\frac{\mathrm{S}_{0} \tau_{\mathrm{s}}}{4 \pi}
$$

and finally a 3D Cartesian distribution function parametrized by the speed $v^{12,20,37,38}$

$$
f_{\mathrm{Car}}^{3 \mathrm{D}}(v)=\frac{\mathrm{S}_{0} \tau_{\mathrm{s}}}{4 \pi} \frac{\mathrm{H}\left(v_{b}-v\right)}{v^{3}+v_{\mathrm{c}}^{3}} .
$$

This representation of the slowing-down distribution is plotted in Fig. 6 for typical ITER parameters.

For fast-ion modelling, it is convenient to express the prefactor $S_{0} \tau_{s} / 4 \pi$ in terms of the fast-ion density $n$. This relation can be established by integrating over $3 \mathrm{D}$ velocity space. As the slowing-down distribution function is isotropic and the variable in Eq. (95) is the speed, the integral is easiest in spherical coordinates. In spherical coordinates, the isotropic slowing-down distribution is

$$
f_{s p h}^{3 \mathrm{D}}(v, \zeta)=\frac{\mathrm{S}_{0} \tau_{s}}{4 \pi} \frac{v^{2} \sin \zeta \mathrm{H}\left(v_{b}-v\right)}{v^{3}+v_{c}^{3}} .
$$

Integration gives

$$
\begin{aligned}
n & =\frac{\mathrm{S}_{0} \tau_{s}}{4 \pi} \int_{0}^{\infty} \int_{0}^{\pi} \int_{0}^{2 \pi} \frac{v^{2} \sin \zeta \mathrm{H}\left(v_{b}-v\right)}{v^{3}+v_{\mathrm{c}}^{3}} d \eta d \zeta d v \\
& =\frac{\mathrm{S}_{0} \tau_{\mathrm{s}}}{4 \pi} \int_{0}^{v_{b}} \frac{4 \pi v^{2}}{v^{3}+v_{\mathrm{c}}^{3}} d v,
\end{aligned}
$$

where we have changed the upper integration limit in $v$ according to the Heaviside function. The remaining integral over $v$ yields the desired relation between the fast-ion density, the source rate, and the slowing-down time

$$
n=\frac{\mathrm{S}_{0} \tau_{\mathrm{s}}}{3} \ln \left(1+\left(\frac{v_{b}}{v_{\mathrm{c}}}\right)^{3}\right) .
$$

The actual time $\tilde{\tau}_{s}$ for a particle to slow down from $v_{b}$ to zero is given by $n=\mathrm{S}_{0} \tilde{\tau}_{s}$ so that

$$
\tilde{\tau}_{\mathrm{s}}=\frac{\tau_{s}}{3} \ln \left(1+\left(\frac{v_{b}}{v_{c}}\right)^{3}\right) .
$$

The isotropic 3D Cartesian slowing-down distribution parametrized by the speed is hence given in terms of the fast-ion density by

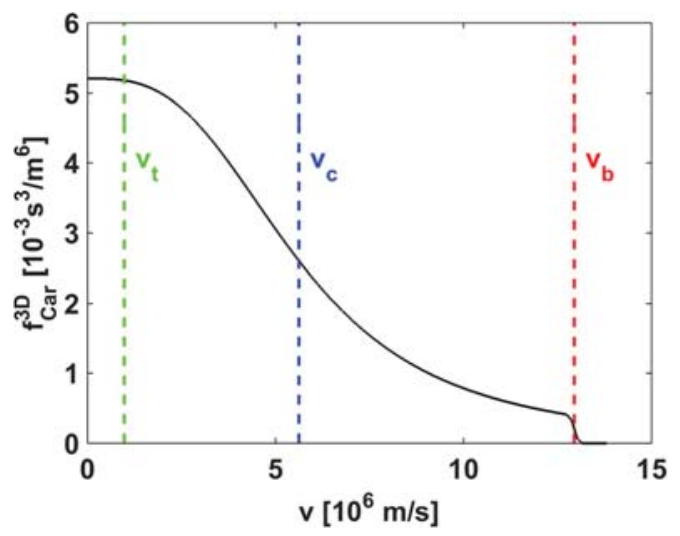

FIG. 6. Slowing-down distribution function $f_{C a r}^{3 D}(v)$ for $\alpha$-particles in ITER. The birth speed, the crossover speed, and the thermal speed are marked. 


$$
f_{\mathrm{C} a r}^{3 \mathrm{D}}(v)=\frac{3 n}{4 \pi \ln \left(1+\left(\frac{v_{b}}{v_{\mathrm{c}}}\right)^{3}\right)} \frac{\mathrm{H}\left(v_{b}-v\right)}{v^{3}+v_{\mathrm{c}}^{3}} .
$$

In 3D Cartesian coordinates $\left(v_{\|}, v_{\perp 1}, v_{\perp 2}\right)$, Eq. (102) becomes

$$
\begin{aligned}
& f_{\mathrm{Car}}^{3 \mathrm{D}}\left(v_{\|}, v_{\perp 1}, v_{\perp 2}\right)=\frac{3 n}{4 \pi \ln \left(1+\left(\frac{v_{b}}{v_{c}}\right)^{3}\right)}
\end{aligned}
$$

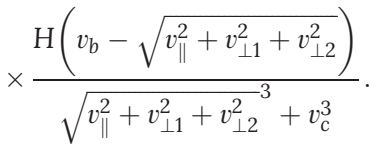

The isotropic 3D Cartesian slowing-down distribution function parametrized by $\left(v_{\|}, v_{\perp}\right)$ is

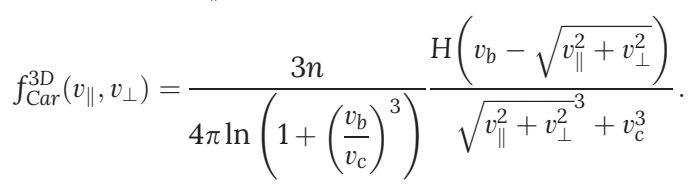

In $2 \mathrm{D}$ Cartesian coordinates $\left(v_{\|}, v_{\perp}\right)$, it is

$$
f_{\text {Car }}^{2 \mathrm{D}}\left(v_{\|}, v_{\perp}\right)=\frac{3 n}{2 \ln \left(1+\left(\frac{v_{b}}{v_{c}}\right)^{3}\right)} \frac{v_{\perp} \mathrm{H}\left(v_{b}-\sqrt{v_{\|}^{2}+v_{\perp}^{2}}\right)}{{\sqrt{v_{\|}^{2}+v_{\perp}^{2}}+v_{c}^{3}}^{3}} .
$$

Transformation of Eq. (105) to (E, $p)$-space gives

$$
f_{\mathrm{Ep}}^{2 \mathrm{D}}(\mathrm{E})=\frac{3 n}{4 \ln \left(1+\left(\frac{E_{b}}{E_{c}}\right)^{3 / 2}\right)} \frac{\sqrt{E} H\left(E_{b}-E\right)}{E^{3 / 2}+E_{c}^{3 / 2}} .
$$

Here, the crossover energy is

$$
\mathrm{E}_{\mathrm{c}}=\frac{1}{2} m_{\alpha} v_{\mathrm{c}}^{2},
$$

which can be expressed in terms of the electron temperature

$$
E_{c}=\left(\left(\frac{3 \sqrt{\pi} Z_{1}}{4}\right)^{2} \frac{m_{\alpha}}{m_{e}}\right)^{1 / 3} \mathrm{~T}_{e} .
$$

For $n_{\mathrm{D}}=n_{\mathrm{T}}=0.5 n_{e}$, we get for the crossover energy of $\alpha$ particles

$$
\mathrm{E}_{\mathrm{c}}=33 \mathrm{~T}_{e} .
$$

In the similar $(v, p)$-space, the isotropic slowing-down distribution reads

$$
f_{v p}^{2 \mathrm{D}}(v)=\frac{3 n}{2 \ln \left(1+\left(\frac{v_{b}}{v_{c}}\right)^{3}\right)} \frac{v^{2} \mathrm{H}\left(v_{b}-v\right)}{v^{3}+v_{c}^{3}} .
$$

The isotropic slowing-down distribution as expected in ITER is plotted in various coordinate systems in Fig. 7.

The 1D speed distribution is obtained by multiplication of Eq. (102) with $4 \pi v^{2}$ according to Eq. (30)

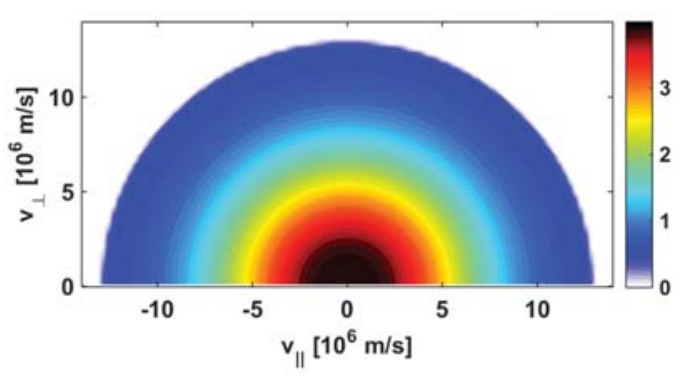

(a) $f_{C a r}^{3 D}\left(v_{\|}, v_{\perp}\right)$

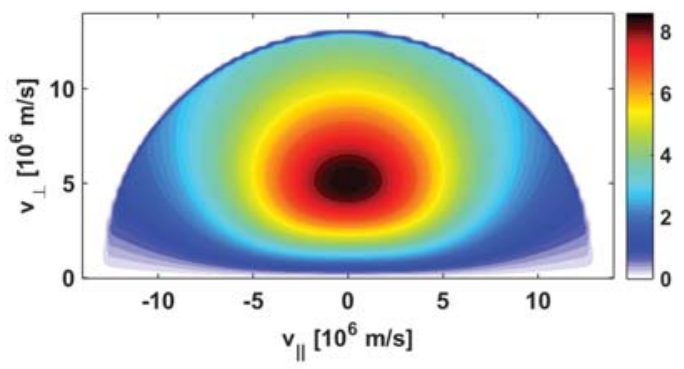

(b) $f_{C a r}^{2 D}\left(v_{\|}, v_{\perp}\right)$

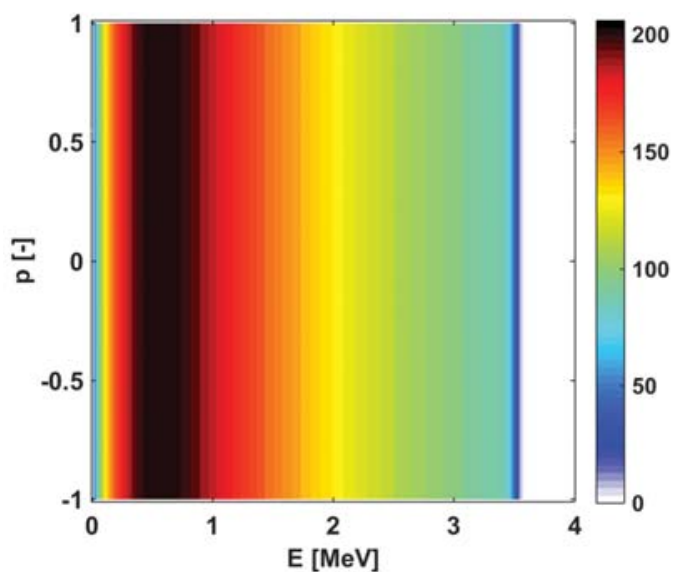

(c) $f_{E p}^{2 D}(E, p)$

FIG. 7. $\alpha$-particle slowing-down velocity distribution function with ITER-relevant parameters in various coordinate systems. No ions above the birth energies are found.

$$
f_{v}^{1 \mathrm{D}}(v)=\frac{3 n}{\ln \left(1+\left(\frac{v_{b}}{v_{c}}\right)^{3}\right)} \frac{v^{2} \mathrm{H}\left(v_{b}-v\right)}{v^{3}+v_{\mathrm{c}}^{3}} .
$$

The energy spectrum of the slowing-down distribution is found by a change in the variable [Eq. (22)]

$$
f_{E}^{1 D}(E)=\frac{3 n}{2 \ln \left(1+\left(\frac{E_{b}}{E_{c}}\right)^{3 / 2}\right)} \frac{\sqrt{E} H\left(E_{b}-E\right)}{E^{3 / 2}+E_{c}^{3 / 2}} .
$$


Figure 8 illustrates the energy spectrum of the isotropic slowing-down distribution expected for ITER. The energy spectra of fusion $\alpha$-particles and other fast ions are measurement requirements of energetic particle diagnostics at ITER. ${ }^{39}$ The energy and speed spectra of the slowing-down distribution [Eqs. (112) and (111)] can also be obtained by integration of Eqs. (106) and (110) over the pitch, respectively, which gives a factor two due to the isotropy. The integral over energy gives the fastion density since

$$
\int_{0}^{E_{b}} \frac{\sqrt{E}}{E^{3 / 2}+E_{c}^{3 / 2}} d E=\frac{2}{3} \ln \left(1+\left(\frac{E_{b}}{E_{c}}\right)^{3 / 2}\right) .
$$

To account for a distribution of birth energies in the lab frame, we can replace the Heaviside function with the complementary error function which does not change the above integral and has the property

$$
\lim _{\Delta E \rightarrow 0} \frac{1}{2} \operatorname{erfc} \frac{E-E_{b}}{\Delta E}=H\left(E_{b}-E\right)
$$

The energy distribution is then

$$
f_{E}^{1 D}(E)=\frac{3 n}{4 \ln \left(1+\left(\frac{E_{b}}{E_{c}}\right)^{3 / 2}\right)} \frac{\sqrt{E}}{E^{3 / 2}+E_{c}^{3 / 2}} \operatorname{erfc} \frac{E-E_{b}}{\Delta E} .
$$

However, we will use the Heaviside function as an ideal model in the following for simplicity.

The $1 \mathrm{D}$ projection of the slowing-down distribution is not as easily computed as for the Maxwellian since the variables are not easily separated. However, we have computed the projection explicitly using the weight function formalism according to Eq. (33). The integral is straightforward in (E, p)-space since the isotropic slowing-down distribution does not depend on the pitch. Thus, we can first integrate just the weight function $w$ over the pitch and then do the integration over the energy. The result is

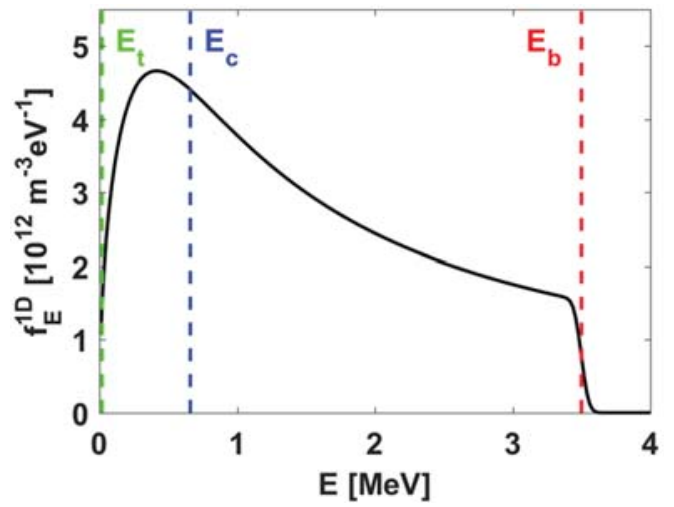

FIG. 8. $\alpha$-particle slowing-down energy distribution function as expected in ITER The energy distribution function is more flat as compared to Fig. 6.

$$
\begin{aligned}
g(u)= & \frac{n}{4 v_{c} \ln \left(1+\left(\frac{v_{b}}{v_{c}}\right)^{3}\right)} \times\left(\ln \left(\left|\frac{v_{b}^{2}-v_{b} v_{c}+v_{c}^{2}}{u^{2}-|u| v_{c}+v_{c}^{2}}\right|\left(\frac{|u|+v_{c}}{v_{b}+v_{c}}\right)^{2}\right)\right. \\
& \left.+2 \sqrt{3}\left(\arctan \frac{2 v_{b}-v_{c}}{\sqrt{3} v_{c}}-\arctan \frac{2|u|-v_{c}}{\sqrt{3} v_{c}}\right)\right) .
\end{aligned}
$$

To our knowledge, Eq. (116) has not been published until now. It is a useful equation for basic modeling of $\alpha$-particle diagnostic in fusion plasmas. The projection does not depend on the angle between the line-of-sight and the magnetic field as we expect for an isotropic function. The $1 \mathrm{D}$ projection of the isotropic slowing-down distribution is illustrated in Fig. 9. Compared to a Maxwellian, the tails are cut off and are elevated. The projected slowing-down function does not have a sudden jump at the birth velocity as the function $f_{\text {Car }}^{3 D}$.

\section{B. Anisotropic slowing-down distribution}

A useful analytical function modeling NBI velocity distribution functions has been found more than 40 years ago. ${ }^{11,12} \mathrm{NBI}$ distribution functions are nowadays routinely computed with several computer codes that take spatial effects into account, ${ }^{40}$ e.g., the TRANSP module NUBEAM ${ }^{16}$ or ASCOT. ${ }^{41}$ It has also recently become possible to measure NBI distribution functions directly by velocity-space tomography. ${ }^{14,42}$ The analytical model of NBI distribution functions described here provides useful guidance to compare the measurements and the simulations with. ${ }^{43}$ The model is found as a steady-state solution of the simplified Fokker-Planck equation

$$
\begin{aligned}
& \frac{1}{\tau_{\mathrm{s}} v^{2}} \frac{\partial}{\partial v}\left(\left(v^{3}+v_{\mathrm{c}}^{3}\right) f_{\mathrm{C} a r}^{3 \mathrm{D}}\right)+\frac{\mathrm{Z}_{2} v_{\mathrm{c}}^{3}}{2 \tau_{\mathrm{s}} v^{3}} \frac{\partial}{\partial p}\left(\left(1-p^{2}\right) \frac{\partial f_{\mathrm{C} a r}^{3 \mathrm{D}}}{\partial p}\right) \\
& \quad+\mathrm{S}=0 .
\end{aligned}
$$

The anisotropic source term S is here taken as the $\delta$-function in speed and a pitch-broadened function $K(p)$ with

$$
\int_{-1}^{1} \mathrm{~K}(p) d p=1
$$

The anisotropic source term is

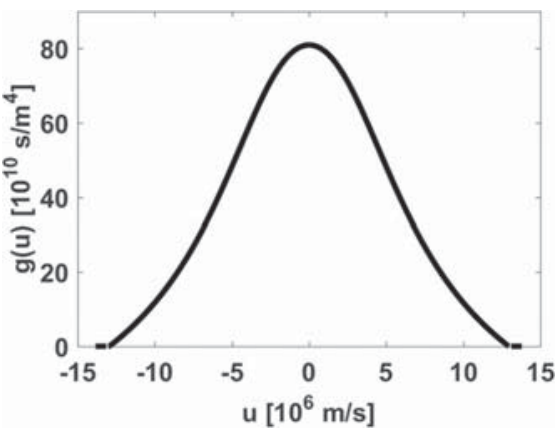

FIG. 9. 1D projection of the isotropic $\alpha$-particle slowing-down distribution function $g(u)$ for any projection angle $\phi$ as expected for ITER. 


$$
\mathrm{S}(v, p)=\frac{\mathrm{S}_{0} \delta\left(v-v_{b}\right) \mathrm{K}(p)}{4 \pi v^{2}} .
$$

The steady-state solution of the Fokker-Planck equation is written as expansion in Legendre polynomials as 3D Cartesian phase-space densities parametrized in $(v, p)$-coordinates

$$
f_{\mathrm{Car}}^{3 \mathrm{D}}(v, p)=\frac{1}{2 \pi} \frac{\mathrm{S}_{0} \tau_{\mathrm{s}}}{v^{3}+v_{\mathrm{c}}^{3}} \sum_{l=0}^{\infty}\left(l+\frac{1}{2}\right) u^{l(l+1)} \mathrm{P}_{l}(p) \mathrm{K}_{l} \mathrm{H}\left(v_{b}-v\right)
$$

or fully transformed to $(v, p)$-coordinates

$$
f_{v p}^{2 \mathrm{D}}(v, p)=\frac{\mathrm{S}_{0} \tau_{\mathrm{s}} v^{2}}{v^{3}+v_{\mathrm{c}}^{3}} \sum_{l=0}^{\infty}\left(l+\frac{1}{2}\right) u^{l(l+1)} \mathrm{P}_{l}(p) \mathrm{K}_{l} \mathrm{H}\left(v_{b}-v\right),
$$

where

$$
\begin{aligned}
& u=\left(\frac{v_{b}^{3}+v_{\mathrm{c}}^{3}}{v^{3}+v_{\mathrm{c}}^{3}} \frac{v^{3}}{v_{b}^{3}}\right)^{\mathrm{Z}_{2} / 6}, \\
& \mathrm{~K}_{l}=\int_{-1}^{1} \mathrm{~K}(p) \mathrm{P}_{l}(p) d p .
\end{aligned}
$$

The Legendre polynomials $P_{l}(p)$ are polynomials of order $l$. The first few Legendre polynomials are

$$
\begin{aligned}
& \mathrm{P}_{0}(p)=1, \quad \mathrm{P}_{1}(p)=p, \\
& \mathrm{P}_{2}(p)=\frac{1}{2}\left(3 p^{2}-1\right), \quad \mathrm{P}_{3}(p)=\frac{1}{2}\left(5 p^{3}-3 p\right) .
\end{aligned}
$$

Higher-order Legendre polynomials can be computed by Bonnet's recursion formula

$$
\mathrm{P}_{l+1}(p)=\frac{2 l+1}{l+1} p \mathrm{P}_{l}(p)-\frac{l}{l+1} \mathrm{P}_{l-1}(p)
$$

As the Legendre polynomials are orthogonal in $[-1 ; 1]$, several important integrals become very simple. The fast-ion density depends only on the first term of the sum in Eq. (121) with $l=0$ since

$$
f_{v p}^{2 \mathrm{D}}(v, p)=\mathrm{P}_{0}(p) f_{v p}^{2 \mathrm{D}}(v, p),
$$

and the integral over pitch is therefore zero for all $l>0$ due to orthogonality.

$$
\begin{aligned}
n & =\int_{0}^{\infty} \int_{-1}^{1} f_{v p}^{2 \mathrm{D}}(v, p) d p d v=\mathrm{S}_{0} \tau_{\mathrm{s}} \int_{0}^{v_{b}} \frac{v^{2}}{v^{3}+v_{\mathrm{c}}^{3}} d v \\
& =\frac{\mathrm{S}_{0} \tau_{s}}{3} \ln \left(1+\left(\frac{v_{b}}{v_{\mathrm{c}}}\right)^{3}\right)
\end{aligned}
$$

This relation is the same as for the isotropic slowing-down distribution [Eq. (100)]. Hence, $S_{0} \tau_{s}$ can be eliminated from the distribution function to express the scaling of the distribution function in terms of the fast-ion density

$$
\begin{aligned}
f_{v p}^{2 \mathrm{D}}(v, p)= & \frac{3 n}{\ln \left(1+\frac{v_{b}^{3}}{v_{c}^{3}}\right)} \frac{v^{2}}{v^{3}+v_{\mathrm{c}}^{3}} \\
& \times \sum_{l=0}^{\infty}\left(l+\frac{1}{2}\right) u^{l(l+1)} \mathrm{P}_{l}(p) \mathrm{K}_{l} \mathrm{H}\left(v_{b}-v\right) .
\end{aligned}
$$

As the $l=0$ term gives $\frac{1}{2}$, this term corresponds to the isotropic slowing down distribution [Eq. (110)].

An example of a typical NBI distribution function with an injection energy of $1 \mathrm{MeV}$, as foreseen in ITER, appears in Fig. 10. ITER has negative-ion sources, and therefore, no injection sources at half- and one-third energy appear. To model a typical NBI distribution function for positive-ion sources, which are typical in present tokamaks, one can sum anisotropic slowing-down functions for the full-, half-, and one-third injection energies. It is straightforward to give analytical expressions for the anisotropic slowing-down distributions in the other coordinate

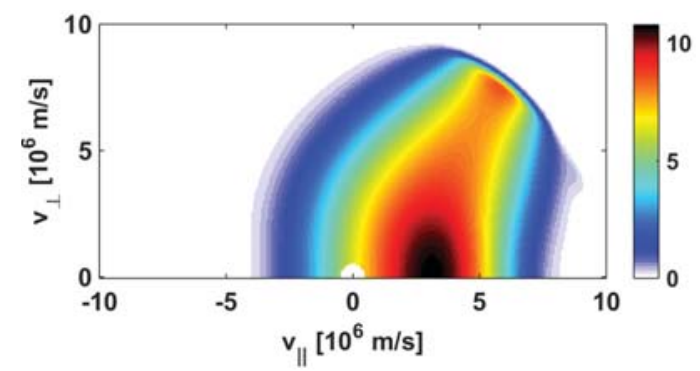

(a) $f_{C a r}^{3 D}\left(v_{\|}, v_{\perp}\right)$

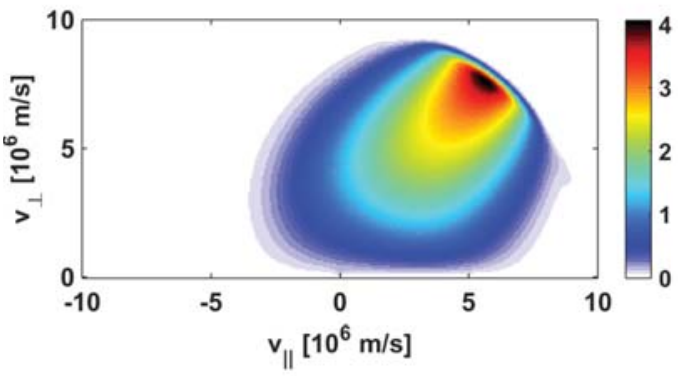

(b) $f_{\text {Car }}^{2 D}\left(v_{\|}, v_{\perp}\right)$

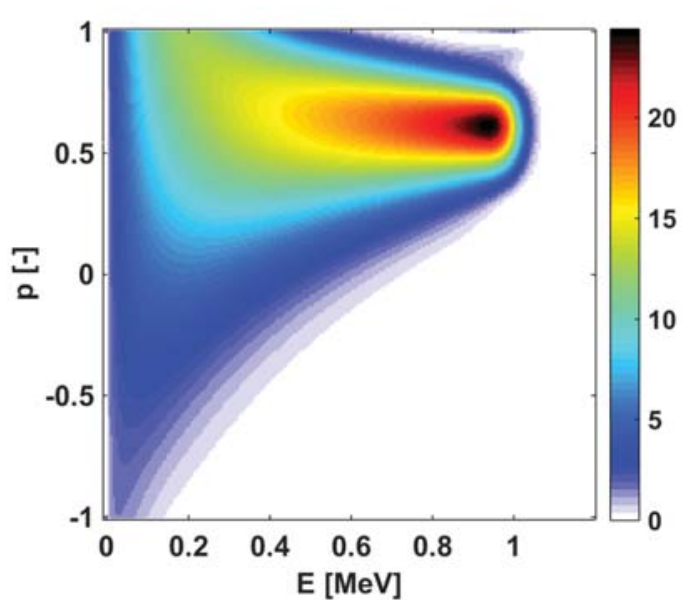

(c) $f_{E p}^{2 D}(E, p)$

FIG. 10. NBI distribution function in various coordinate systems showing the slowing down and pitch angle scattering of a particle source at an energy of $1 \mathrm{MeV}$ at a pitch of 0.6 . 
systems. However, the pitch coordinate is essential for the use of Legendre polynomials so that the expressions are by far simplest when the pitch is used as a coordinate. Therefore, we do not give explicit expressions in the other coordinate systems and transform the function numerically in Fig. 10.

Finally, the 1D projection of NBI distribution functions along the line-of-sight plays a prominent role in fast-ion diagnostics and strongly influences the basic shape of the observable spectra. $1 \mathrm{D}$ projections along several directions of the line-of-sight are illustrated in Fig. 11. For the given highly anisotropic NBI distribution function in Fig. 10, the measurable spectrum can be symmetric or asymmetric with bias towards redshift or blueshift to varying degrees depending on the geometry of the line-of-sight. Analytical formulas for the projection of this distribution have been computed for the first ten Legendre polynomials. However, as the analytical expressions become fairly cluttered, it seems at this point more convenient to compute projections numerically by the formalism for arbitrary distribution functions. ${ }^{34}$

\section{PHASE-SPACE DISTRIBUTION FUNCTIONS}

The velocity distribution functions discussed in this tutorial describe the velocity distribution at a single point in the plasma. In tokamaks, the full phase-space distribution function can be described by three constants of motion which are the energy, the magnetic moment $\mu$, and the canonical toroidal angular momentum $\mathrm{P}_{\phi}$ (Ref. 37)

$$
\begin{gathered}
\mathrm{E}=\frac{1}{2} m v^{2}, \\
\mu=\frac{m v_{\perp}^{2}}{2 \mathrm{~B}}, \\
\mathrm{P}_{\phi}=m \mathrm{R} \frac{\mathrm{B}_{\phi}}{\mathrm{B}} v_{\|}-q \Psi,
\end{gathered}
$$

where $\mathrm{R}$ is the major radius coordinate, $\mathrm{B}_{\phi}$ is the toroidal magnetic field component, and $\Psi$ is the poloidal magnetic flux. The phase-space distribution function $f_{\mathbf{x v}}^{6 \mathrm{D}}\left(\mathrm{E}, \mu, \mathrm{P}_{\phi}, \sigma\right)$ completely describes all ions in the plasma. The binary parameter $\sigma$ $=\operatorname{sgn}\left(v_{\|}\right)$labels if a passing orbit is co-going or counter-going.

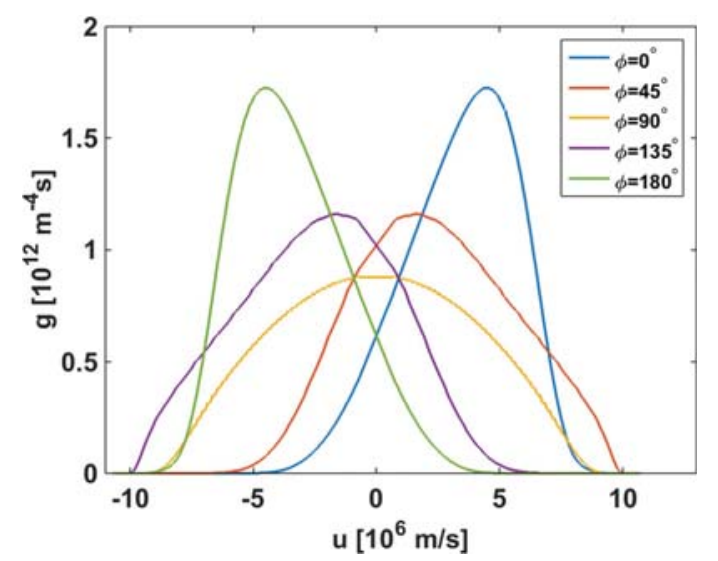

FIG. 11. 1D projections of the NBI distribution function from Fig. 10 for various projection angles $\phi$.
This label is necessary since co- and counter-going orbits with the same $\left(\mathrm{E}, \mu, \mathrm{P}_{\phi}\right)$ exist. One can thus consider this distribution function as to be composed of two 3D distribution functions. It should be noted that the particle number in a phase-space volume element is usually

$$
d \mathrm{~N}=f_{\mathbf{x v}}^{6 \mathrm{D}}\left(\mathrm{E}(\mathbf{x}, \mathbf{v}), \mu(\mathbf{x}, \mathbf{v}), \mathrm{P}_{\phi}(\mathbf{x}, \mathbf{v}), \sigma\right) d \mathbf{x} d \mathbf{v}
$$

The mixing of position space and velocity space makes it difficult to give analytical models for this distribution. Nevertheless, the phase-space distribution is highly important for stability calculations, and it could be possible to measure it in the future. ${ }^{44}$ To have a simple analytical model, one can assume the distribution to be separable according to ${ }^{45}$

$$
f_{\mathbf{x v}}^{6 \mathrm{D}}\left(\mathrm{E}, \mu, \mathrm{P}_{\phi}, \sigma\right)=g_{1}(\mathrm{E}) g_{2}(\mu) g_{3}\left(\mathrm{P}_{\phi}\right) .
$$

Then, physical models for the energy distribution can be assumed, e.g., the slowing-down distribution or a ring distribution. The magnetic topology in tokamaks makes it difficult to give general analytical models for $\mu$ and $\mathrm{P}_{\phi}$. As no complete analytical models exist to our knowledge, we do not discuss phase-space distribution functions parameterized in constants of motion further. In the 3D magnetic field of stellarators, parameterization of the phase-space in three constants of motion is not possible.

\section{CONCLUSIONS}

We have discussed analytical models for several velocity distribution functions: the Maxwellian, the drifting biMaxwellian, the ring distribution, and the isotropic and the anisotropic slowing-down distributions. The ordinary Maxwellian, which is well-known to most physicists, served to give a practical example of a velocity distribution function in the most common coordinate systems used to describe plasmas in magnetic fusion devices: 3D Cartesian, spherical and cylindrical coordinates, 2D Cartesian, $(E, p)$ and $(v, p)-$ coordinates, and 1D speed or energy coordinates. The 1D projection of the distribution functions provides insights into measurements relying on the Doppler shift, e.g., $\mathrm{D}_{\alpha}$-spectroscopy. These distributions and coordinate systems are common and useful for basic plasma modelling, for example, of the bulk plasma or fast-ion velocity distributions from NBI, ICRF heating, or fusion reactions. The analytical models discussed here provide insight into the basic shapes of these distribution functions and allow quick parametric studies and diagnostic design. Our examples are illustrated for ITERrelevant parameters. Furthermore, they can serve as guidance to interpret simulations and measurements of velocity distribution functions and their lowest moments: the density, the drift velocity, and the temperature.

\section{ACKNOWLEDGMENTS}

We thank the ITPA Energetic Particle Physics Topical Group for its support.

\section{REFERENCES}

${ }^{7}$ R. P. Feynman, R. B. Leighton, and M. Sands, The Feynman Lectures on Physics (Caltech, 1963). 
${ }^{2}$ L. D. Landau and E. M. Lifshitz, Statistical Physics, 3rd ed. (ButterworthHeinemann, 1980), Vol. 5.

${ }^{3}$ K. S. Thorne and R. D. Blandford, Modern Classical Physics (Princeton University Press, 2017).

${ }^{4}$ J. C. Maxwell, Philos. Mag. 19, 19 (1860).

${ }^{5}$ J. C. Maxwell, Philos. Trans. 157, 49 (1867).

${ }^{6}$ L. Boltzmann, Wien. Ber. 66, 275 (1872).

${ }^{7}$ L. Boltzmann, Wien. Ber. 76, 373 (1877).

${ }^{8}$ L. Spitzer, Physics of Fully Ionized Gases (Interscience, New York, 1956).

${ }^{9}$ D. V. Sivukhin, Rev. Plasma Phys. 4, 93 (1966).

${ }^{10}$ T. H. Stix, Plasma Phys. 14, 367 (1972).

"1J. G. Cordey and W. G. F. Core, Phys. Fluids 17, 1626 (1974).

12J. D. Gaffey, J. Plasma Phys. 16, 149 (1976).

${ }^{13}$ T. H. Stix, Nucl. Fusion 15, 737 (1975).

${ }^{14}$ M. Salewski, B. Geiger, A. Jacobsen, M. García-Muñoz, W. Heidbrink, S. Korsholm, F. Leipold, J. Madsen, D. Moseev, S. Nielsen, J. Rasmussen, M. Stejner, G. Tardini, M. Weiland, and ASDEX Upgrade Team, Nucl. Fusion 54, 023005 (2014)

${ }^{15}$ M. Salewski, M. Nocente, A. Jacobsen, F. Binda, C. Cazzaniga, G. Ericsson, J. Eriksson, G. Gorini, C. Hellesen, A. Hjalmarsson, V. Kiptily, T. Koskela, S. Korsholm, T. Kurki-Suonio, F. Leipold, J. Madsen, D. Moseev, S. Nielsen, J. Rasmussen, M. Schneider, S. Sharapov, M. Stejner, M. Tardocchi, and J. Contributors, Nucl. Fusion 57, 056001 (2017).

${ }^{16}$ A. Pankin, D. McCune, R. Andre, G. Bateman, and A. Kritz, Comput. Phys. Commun. 159, 157 (2004).

${ }^{17}$ D. Moseev, M. Salewski, B. Geiger, M. Garcia-Munoz, and M. Nocente, Rev. Mod. Plasma Phys. 2, 7 (2018).

${ }^{18}$ W. W. Heidbrink, Y. Luo, K. H. Burrell, R. W. Harvey, R. I. Pinsker, and E. Ruskov, Plasma Phys. Controlled Fusion 49, 1457 (2007).

${ }^{19} \mathrm{M}$. Salewski, S. Nielsen, H. Bindslev, V. Furtula, N. Gorelenkov, S. Korsholm, F. Leipold, F. Meo, P. Michelsen, D. Moseev, and M. Stejner, Nucl. Fusion 51, 083014 (2011)

${ }^{20}$ R. J. Goldston and P. H. Rutherford, Introduction to Plasma Physics (IOP Publishing, 1995).

${ }^{21}$ R. O. Dendy, R. J. Hastie, K. G. McClements, and T. J. Martin, Phys. Plasmas 2, 1623 (1995).

${ }^{22}$ W. Cooper, J. Graves, S. Hirshman, T. Yamaguchi, Y. Narushima, S. Okamura, S. Sakakibara, C. Suzuki, K. Watanabe, H. Yamada, and K. Yamazaki, Nucl. Fusion 46, 683 (2006).

${ }^{23}$ S.-R. Huh, N.-K. Kim, B.-K. Jung, K.-J. Chung, Y.-S. Hwang, and G.-H. Kim, Phys. Plasmas 22, 033506 (2015).

${ }^{24}$ P. H. Yoon, Phys. Plasmas 23, 072114 (2016).

${ }^{25}$ M. Salewski, B. Geiger, A. Jacobsen, I. Abramovic, S. Korsholm, F. Leipold, B. Madsen, J. Madsen, R. McDermott, D. Moseev, S. Nielsen, M. Nocente, J. Rasmussen, M. Stejner, M. Weiland, T. E. M. Team, and T. A. U. Team, Nucl. Fusion 58, 036017 (2018).

${ }^{26} \mathrm{M}$. Vandas and P. Hellinger, Phys. Plasmas 22, 062107 (2015).

${ }^{27}$ J. Sun, X. Gao, L. Chen, Q. Lu, X. Tao, and S. Wang, Phys. Plasmas 23, 022901 (2016).
${ }^{28}$ C. Hellesen, M. G. Johnson, E. A. Sundén, S. Conroy, G. Ericsson, J. Eriksson, H. Sjöstrand, M. Weiszflog, T. Johnson, G. Gorini, M. Nocente, M. Tardocchi, V. Kiptily, S. Pinches, S. Sharapov, and J. E. Contributors, Nucl. Fusion 53, 113009 (2013).

${ }^{29}$ D. Winske, C. S. Wu, Y. Y. Li, Z. Z. Mou, and S. Y. Guo, J. Geophys. Res.: Space Phys. 90, 2713, https://doi.org/10.1029/JA090iA03p02713 (1985).

${ }^{30}$ V. Florinski, G. P. Zank, J. Heerikhuisen, Q. Hu, and I. Khazanov, Astrophys. J. 719, 1097 (2010).

${ }^{31}$ T. Umeda, S. Matsukiyo, T. Amano, and Y. Miyoshi, Phys. Plasmas 19, 072107 (2012)

${ }^{32}$ E. J. Summerlin, A. F. Vinas, T. E. Moore, E. R. Christian, and J. F. Cooper, Astrophys. J. 793, 93 (2014).

${ }^{33}$ F. Hadi, P. H. Yoon, and A. Qamar, Phys. Plasmas 22, 022112 (2015).

${ }^{34} \mathrm{M}$. Salewski, B. Geiger, D. Moseev, W. W. Heidbrink, A. S. Jacobsen, S. B. Korsholm, F. Leipold, J. Madsen, S. K. Nielsen, J. Rasmussen, M. Stejner, M. Weiland, and ASDEX Upgrade Team, Plasma Phys. Controlled Fusion 56, 105005 (2014).

${ }^{35}$ H. ITER Physics Expert Group on Energetic Particles, C. Drive, and I. P. B. Editors, Nucl. Fusion 39, 2471 (1999).

${ }^{36}$ M. Salewski, M. Nocente, B. Madsen, I. Abramovic, M. Fitzgerald, G. Gorini, P. Hansen, W. Heidbrink, A. Jacobsen, T. Jensen, V. Kiptily, E. Klinkby, S. Korsholm, T. Kurki-Suonio, A. Larsen, F. Leipold, D. Moseev, S. Nielsen, S. Pinches, J. Rasmussen, M. Rebai, M. Schneider, A. Shevelev, S. Sipilä, M. Stejner, and M. Tardocchi, Nucl. Fusion 58, 096019 (2018).

${ }^{37}$ P. Helander and D. J. Sigmar, Collisional Transport in Magnetized Plasmas (Cambridge University Press, 2002).

${ }^{38}$ C. Angiono and A. G. Peeters, Phys. Plasmas 15, 052307 (2008).

${ }^{39}$ A. Donne, A. Costley, R. Barnsley, H. Bindslev, R. Boivin, G. Conway, R. Fisher, R. Giannella, H. Hartfuss, M. von Hellermann, E. Hodgson, L. Ingesson, K. Itami, D. Johnson, Y. Kawano, T. Kondoh, A. Krasilnikov, Y. Kusama, A. Litnovsky, P. Lotte, P. Nielsen, T. Nishitani, F. Orsitto, B. Peterson, G. Razdobarin, J. Sanchez, M. Sasao, T. Sugie, G. Vayakis, V. Voitsenya, K. Vukolov, C. Walker, K. Young, and ITPA Topical Group on Diagnostics, Nucl. Fusion 47, S337 (2007).

${ }^{40}$ R. Goldston, D. McCune, H. Towner, S. Davis, R. Hawryluk, and G. Schmidt, J. Comput. Phys. 43, 61 (1981).

${ }^{41}$ E. Hirvijoki, O. Asunta, T. Koskela, T. Kurki-Suonio, J. Miettunen, S. Sipilä, A. Snicker, and S. Äkäslompolo, Comput. Phys. Commun. 185, 1310 (2014)

${ }^{42}$ M. Salewski, M. Nocente, G. Gorini, A. Jacobsen, V. Kiptily, S. Korsholm, F. Leipold, J. Madsen, D. Moseev, S. Nielsen, J. Rasmussen, M. Stejner, M. Tardocchi, and J. Contributors, Nucl. Fusion 56, 046009 (2016).

${ }^{43}$ M. Weiland, R. Bilato, R. Dux, B. Geiger, A. Lebschy, F. Felici, R. Fischer, D. Rittich, and M. van Zeeland, ASDEX Upgrade Team, and Eurofusion MST1 Team, Nucl. Fusion 58, 082032 (2018).

${ }^{44}$ L. Stagner and W. W. Heidbrink, Phys. Plasmas 24, 092505 (2017).

${ }^{45}$ S. Pinches, L. Appel, J. Candy, S. Sharapov, H. Berk, D. Borba, B. Breizman, T. Hender, K. Hopcraft, G. Huysmans, and W. Kerner, Comput. Phys. Commun. 111, 133 (1998). 